\title{
Magnetic flux emergence in granular convection: radiative MHD simulations and observational signatures ${ }^{\star}$
}

\author{
M. C. M. Cheung ${ }^{1, \star \star}$, M. Schüssler ${ }^{1}$, and F. Moreno-Insertis ${ }^{2,3}$
}

\author{
1 Max Planck Institute for Solar System Research, 37191 Katlenburg-Lindau, Germany \\ e-mail: [cheung,msch] @mps.mpg.de; cheung@lmsal.com \\ 2 Instituto de Astrofísica de Canarias, 38200 La Laguna (Tenerife), Spain \\ e-mail: fmi@ll.iac.es \\ 3 Dept of Astrophysics, Faculty of Physics, University of La Laguna, 38200 La Laguna (Tenerife), Spain
}

Received 2 January 2007 / Accepted 19 February 2007

\section{ABSTRACT}

\begin{abstract}
Aims. We study the emergence of magnetic flux from the near-surface layers of the solar convection zone into the photosphere. Methods. To model magnetic flux emergence, we carried out a set of numerical radiative magnetohydrodynamics simulations. Our simulations take into account the effects of compressibility, energy exchange via radiative transfer, and partial ionization in the equation of state. All these physical ingredients are essential for a proper treatment of the problem. Furthermore, the inclusion of radiative transfer allows us to directly compare the simulation results with actual observations of emerging flux.

Results. We find that the interaction between the magnetic flux tube and the external flow field has an important influence on the emergent morphology of the magnetic field. Depending on the initial properties of the flux tube (e.g. field strength, twist, entropy etc.), the emergence process can also modify the local granulation pattern. The emergence of magnetic flux tubes with a flux of $10^{19} \mathrm{Mx}$ disturbs the granulation and leads to the transient appearance of a dark lane, which is coincident with upflowing material. These results are consistent with observed properties of emerging magnetic flux.
\end{abstract}

Key words. Sun: photosphere - Sun: magnetic fields - Sun: atmosphere - Sun: granulation - magnetohydrodynamics (MHD) radiative transfer

\section{Introduction}

Solar magnetic fields on the surface of the Sun exist and evolve over a wide range of length- and time-scales. The most prominent magnetic features on the solar surface are sunspots. In addition, there is a whole hierarchy of magnetic features including pores, micropores, plages and faculae. An active region is an extended bipolar configuration on the solar surface resulting from the emergence of magnetic fields from the convection zone (Parker 1955). In terms of the amount of magnetic flux in each polarity, there is a continuous spectrum of active region sizes (Hagenaar et al. 2003). Although a partitioning of the flux spectrum for the sake of classification may seem somewhat arbitrary, it allows us to conveniently refer to active regions of different sizes. Large active regions have polarities containing a flux of $>5 \times 10^{21} \mathrm{Mx}$ and contain sunspots. In large active regions, the magnetic flux is shared amongst the whole spectrum of magnetic features. Large active regions have lifetimes of up to months. Small active regions, which contain a flux of $1 \times 10^{20}-5 \times$ $10^{21} \mathrm{Mx}$ in each polarity, may consist of pores and smaller magnetic features but lack sunspots. Small active regions may persist for up to days to weeks. Ephemeral active regions have even less flux $\left(3 \times 10^{18}-1 \times 10^{20} \mathrm{Mx}\right)$, and have lifetimes of only hours to days (Zwaan 1987). Often, ephemeral active regions are simply referred to as ephemeral regions.

\footnotetext{
* Movies are only available in electronic form at http://www. aanda.org

$\star \star$ Present address: Lockheed Martin Solar and Astrophysics Laboratory, Bldg/252 3251 Hanover St., Palo Alto, CA 94304, USA.
}

The characteristic timescale for the emergence of flux is typically only a small fraction of the lifetime of an active region. For instance, almost all the flux of a large active region emerges within the first $\sim 4$ days of their development (Zwaan 1985; Hagenaar 2001). Observations of the birth of an active region (AR) indicate that the total flux in each polarity of the AR is not the consequence of the emergence of a coherent, monolithic flux bundle. Rather, it builds up as the result of many small flux bundles emerging simultaneously or in succession. An Emerging Flux Region (EFR) is the area on the solar surface where these emergence events take place (Zirin 1972; Zwaan 1985). The onset of the birth of an active region is characterized by the appearance of a compact and very bright plage. The plage consists of magnetic flux elements of opposite polarity, which move apart at an initial velocity of about $2 \mathrm{~km} \mathrm{~s}^{-1}$. New flux continues to emerge near the polarity inversion line. The orientation of the emerging field is not random. Rather, it is roughly aligned along the axis connecting the two polarities. As a result of emergence, flux is accumulated in both polarities. If sufficient flux has emerged, pores and possibly sunspots appear. These tend to be formed near the leading and following edges of the expanding plage (see Zwaan 1985, and references therein).

The granulation pattern in an EFR may appear different than that of the quiet Sun. In quiet-Sun granulation, bright granules correspond to upflows whereas the dark intergranular boundaries consist of downflow lanes and vertices. Transient dark alignments in the central part of an EFR have been detected in continuum images and also in maps of the core intensity of photospheric spectral lines (Bray et al. 1984; Brants \& Steenbeek 1985; Zwaan 1985; Strous \& Zwaan 1999). The darkenings are 
roughly aligned along the axis connecting the two polarities of the active region and typically last about $10 \mathrm{~min}$. In the continuum, they are darker than the intergranular boundaries but the spectral lines show Doppler shifts corresponding to upward velocities exceeding $0.5 \mathrm{~km} \mathrm{~s}^{-1}$. By estimating the diameter and strength of emerging flux bundles that lead to the appearance of dark alignments in an EFR, Brants \& Steenbeek (1985) estimated that each bundle contains a longitudinal flux of about $10^{19} \mathrm{Mx}$. This value is consistent with the estimate given by Born (1974), who measured the total flux of an active region and counted the number of arch filaments in $\mathrm{H} \alpha$.

On the basis of the observations previously summarized, Zwaan (1978, 1985) constructed a heuristic model of an EFR at and below the solar surface. In his scenario, flux emerges as a collection of arched flux tubes rising through the convection zone. Deep in the convection zone, the flux tubes connect to an underlying coherent large flux tube. Near the surface, however, they are separated from each other. In his model, the transient dark alignments in the observations are locations of the apices of arched flux tubes emerging at the surface, which appear dark because the horizontal magnetic field suppresses turbulent heat exchange. After the horizontal top of a tube has emerged, the photospheric footpoints of the tubes separate and the field at the footpoints become increasingly vertical. The coalescence of the vertical flux elements in each polarity may lead to the formation of pores and sunspots if sufficient flux is accumulated.

Subsequent observations of EFRs are consistent with various aspects of Zwaan's heuristic model. Strous \& Zwaan (1999) performed a statistical analysis of over two hundred emergence events in a single EFR. They found that emerging flux is characterized by the transient appearance of dark alignments between the polarities. Often, they observed the appearance of faculae at the ends of the dark alignments. The faculae are typically associated with downflows. More recently, Lites et al. (1998) and Kubo et al. (2003) have carried out observations of the full Stokes vector in emerging flux regions and report the detection of horizontal fields with strengths of about 200-600 G. As the newly emerged flux moves away from the emergence site, the fields become vertical and obtain strengths in excess of $1000 \mathrm{G}$.

The aim of this study is to understand the process of magnetic flux emergence from the convection zone into the photosphere by means of numerical simulations. At present, it is not computationally feasible to carry out numerical simulations that include all the relevant physics for the emergence of magnetic flux through all layers of the solar convection zone and atmosphere. To progress, different aspects of the problem have to be addressed separately.

One line of previous research has focused on studying the rise of buoyant magnetic flux tubes in a stratified layer by means of 2D and 3D MHD simulations. Most of these simulations (see, for example, Schüssler 1979; Moreno-Insertis \& Emonet 1996; Longcope et al. 1996; Emonet \& Moreno-Insertis 1998; Fan et al. 1998; Cheung et al. 2006) start from a buoyant magnetic flux tube embedded in an initially static atmosphere. Dorch et al. (2001) was the first to study the interaction of magnetic flux tubes with convective flows. They found that the convective motion of the flow external to the tube enhances the loss rate of flux from the tube. Fan et al. (2003) and Abbett et al. (2000) have also studied the interaction of magnetic flux tubes and convective flow. They found that flux tubes with field strengths exceeding the equipartition value with respect to the kinetic energy density of the convective motions are able to resist deformation by the convective flow, while significantly weaker flux tubes are severely distorted.
Another line of work has focused on studying the dynamics of toroidal magnetic flux tubes rising in the solar convection zone. Numerical simulations of rising toroidal flux tubes under the thin flux tube approximation (Roberts \& Webb 1978; Spruit 1981) have been successful at reproducing the large-scale properties of active regions, such as their emergence latitudes, their tilt angles as well as the asymmetries between leading and following polarities (D'Silva \& Choudhuri 1993; Fan et al. 1993, 1994; Caligari et al. 1995, 1998). When the apices of magnetic loops reach a depth of about 10-20 Mm below the photosphere, their cross-sections have expanded so much that the thin flux tube approximation no longer applies. This limitation means that fully multi-dimensional MHD simulations must be carried out in order to study the details of the emergence process.

Beginning with the work of Forbes \& Priest (1984) and Shibata et al. (1989), a variety of flux emergence simulations have been carried out. The focus of most studies in the literature has been on the evolution of the emerging magnetic field in the corona (Fan 2001; Magara 2001; Magara \& Longcope 2003; Fan \& Gibson 2004; Abbett \& Fisher 2003; Archontis et al. 2004, 2005; Török \& Kliem 2005; Isobe et al. 2005; Galsgaard et al. 2005; Leake \& Arber 2006; Murray et al. 2006; Galsgaard et al. 2007). Recently, Magara (2006) carried out MHD simulations of flux emergence to study the evolution of the coronal and photospheric field of an emerging bipolar region. Like the aforementioned references, his work is relevant for the large scale behaviour of active regions and ignores the effects of convection as well as radiative energy exchange.

In this study, we examine in detail the effects of convective flows and radiative transfer on emerging magnetic flux. To this end, we restrict our attention to flux emergence from the nearsurface part of the convection zone into the photosphere. The article is structured as follows. In Sect. 2, we present the system of radiative MHD equations, the numerical methods used to solve this system as well as the setup of the simulations. In Sect. 3, we discuss the dynamics of rising flux tubes with special emphasis on their interaction with the convective motions. In Sect. 4, we discuss the observational signatures of emerging flux as obtained from our simulations and compare them with observational studies in the literature. Finally, we close the paper with a discussion in Sect. 5.

\section{Governing equations, numerical methods and simulation setup}

\subsection{Governing equations}

The system of radiative MHD equations (in Gaussian cgs units) consists of

$$
\begin{aligned}
& \frac{\partial \varrho}{\partial t}+\nabla \cdot(\varrho \boldsymbol{v})=0, \\
& \frac{\partial \varrho \boldsymbol{v}}{\partial t}+\nabla \cdot\left[\varrho \boldsymbol{v} \otimes \boldsymbol{v}+p_{\mathrm{tot}} \underline{\underline{1}}-\frac{\boldsymbol{B} \otimes \boldsymbol{B}}{4 \pi}\right]=\varrho \boldsymbol{g}+\nabla \cdot \underline{\underline{\tau},} \\
& \frac{\partial e}{\partial t}+\nabla \cdot\left[\boldsymbol{v}\left(e+p_{\mathrm{tot}}\right)-\frac{1}{4 \pi} \boldsymbol{B}(\boldsymbol{v} \cdot \boldsymbol{B})\right]=\varrho(\boldsymbol{g} \cdot \boldsymbol{v}) \\
& \quad+Q_{\mathrm{rad}}+\frac{1}{4 \pi} \nabla \cdot(\boldsymbol{B} \times \eta \nabla \times \boldsymbol{B})+\nabla \cdot(\boldsymbol{v} \cdot \underline{\underline{\tau}})+\nabla \cdot(K \nabla T) \\
& \frac{\partial \boldsymbol{B}}{\partial t}+\nabla \cdot[\boldsymbol{v} \otimes \boldsymbol{B}-\boldsymbol{B} \otimes \boldsymbol{v}]=-\nabla \times(\eta \nabla \times \boldsymbol{B}),
\end{aligned}
$$

where $\varrho$ is the fluid mass density, $\boldsymbol{v}$ the fluid velocity, $\boldsymbol{B}$ the magnetic field, $p_{\text {tot }}=p+|\boldsymbol{B}|^{2} / 8 \pi$ the sum of the gas and magnetic 
pressures, and $e=\varrho \varepsilon+\frac{1}{2} \rho|\boldsymbol{v}|^{2}+|\boldsymbol{B}|^{2} / 8 \pi$ the sum of the internal, kinetic and magnetic energy densities per unit volume. The symbol $\otimes$ denotes a tensor product and $\underline{\underline{1}}$ is the $3 \times 3$ identity matrix. $T$ is the gas temperature and $K$ the thermal conductivity. $\underline{\underline{\tau}}$ represents the viscous stress tensor. $Q_{\text {rad }}$ is the radiative heating rate and $\boldsymbol{g}=-2.74 \times 10^{4} \hat{z} \mathrm{~cm} \mathrm{~s}^{-2}$ is the gravitational acceleration.

Equations (1)-(3) express, respectively, the principles of mass conservation, momentum balance and energy conservation, while Eq. (4) is the induction equation, which describes the evolution of a magnetic field in the plasma. In this equation, $\eta=c^{2} / 4 \pi \sigma$ is the magnetic diffusivity, $c$ the speed of light and $\sigma$ the electrical conductivity.

The MURaM code was used to carry out the radiative MHD simulations in this study. The simulations presented here used grey radiative transfer (i.e. 1 frequency bin). For details about the numerical methods implemented in MURaM, we refer the reader to Vögler (2003) and Vögler et al. (2005).

\subsection{Boundary conditions}

Periodic boundary conditions are imposed at the vertical boundaries. The open boundary condition at the lower boundary is identical to the one used by Vögler et al. (2005).

The top boundary condition has been modified to allow for the advection of magnetic field through the top boundary. This is important because we do not want the emerged magnetic field to be artificially trapped in the photosphere. In principle, the following conditions

$\frac{\partial v_{x}}{\partial z}=\frac{\partial v_{y}}{\partial z}=\frac{\partial v_{z}}{\partial z}=0$,

should suffice for a smooth outflow. This condition, however, is independent of whether the mass flux through the upper boundary is appropriate or unrealistically high. In order to keep the mass flux at appropriate levels, additional constraints must be applied. To this end, we follow Stein \& Nordlund (1998) and implement a fiducial layer above the top boundary of our simulation domain.

The magnetic field above the upper boundary of the domain is matched to a potential field. This requires an extrapolation of the magnetic field in the uppermost domain layer into the ghost cells at each time step. For further details, we refer the reader to Cheung (2006).

\subsection{Initial conditions}

We have carried out a number of simulation runs, each modelling the emergence of an individual flux tube initially embedded in the near-surface layers of the convection zone. In order to prepare the initial condition for the flux emergence simulations, a 3D dynamic model of the near-surface layers of the convection zone and photosphere is required. We obtained such a model by means of a non-magnetic simulation with the MURaM code. The details of the corresponding simulation setup are given in Cheung et al. (2007).

The simulation domain of our model atmosphere has dimensions $24 \times 12 \times 2.3 \mathrm{Mm}^{3}$. The level $z=0$ corresponds to the mean geometrical height where the continuum optical depth at $5000 \AA$ is unity (i.e. $\tau_{5000}=1$ ) and is located at a height of $1.85 \mathrm{Mm}$ above the bottom boundary of the domain. We implant a horizontal, twisted magnetic flux tube with the axis of the tube located at $[y, z]=[6,-1.35] \mathrm{Mm}$ into a snapshot of the 3D hydrodynamic simulation. In all subsequent discussion, $t=0$ refers to the moment when the flux tube is introduced. The longitudinal and transverse (azimuthal) components of the magnetic field have the form

$B_{l}(r)=B_{0} \exp \left(-r^{2} / R_{0}^{2}\right)$

$B_{\theta}(r)=\frac{\lambda r}{R_{0}} B_{l}$

where $r \in\left[0,2 R_{0}\right]$ is the radial distance from the tube axis. The longitudinal flux carried by the tube is $\Phi_{0}=\int B_{l} \mathrm{~d} S \simeq$ $0.98 \pi R_{0}^{2} B_{0}$, so that $R_{0}$ can be viewed as a characteristic "tube radius". The dimensionless twist parameter $\lambda$ specifies the relative strength of the transverse component of the magnetic field with respect to the longitudinal component.

Let $\boldsymbol{v}_{\text {orig }}$ be the original velocity distribution in the nonmagnetic domain. In order to start with an almost stationary flux tube without excessively perturbing the convective flow, we impose the following initial velocity distribution within the tube: $\boldsymbol{v}_{\text {tube }}(r)=\left(1-\mathrm{e}^{-r^{2} / R_{0}^{2}}\right) \boldsymbol{v}_{\text {orig. }}$. With increasing radial distance from the axis, the fluid velocity approaches the original velocity distribution of the external convecting flow.

For a given magnetic field distribution in the tube, the internal gas pressure distribution is specified by requiring that the divergence of the combined stress tensor (gas pressure + Maxwell) be identical to that in the non-magnetic domain (the divergence of the viscous stress tensor is not identical because we have modified the velocity distribution in the magnetic flux tube). Having done so, one is still free to choose the distribution in the tube of one of the following thermodynamic properties: mass density, specific entropy, or temperature. In all of the simulations presented in this paper, the initial flux tube was imparted with a uniform specific entropy distribution $\left(r \leq 2 R_{0}\right)$. The value of the specific entropy was chosen as its average value in the convective upflows at the initial depth of the tube.

\section{Dynamics of subsurface rise and appearance at the photosphere}

\subsection{The rise of a buoyant magnetic flux tube in the presence of convective flows}

Convection plays an important role in determining the properties of emerging flux. Near-surface convection in the Sun consists of disjointed upwellings separated by a network of downflow lanes and vertices (Stein \& Nordlund 1989). An initially horizontally flux tube embedded in this setting will encounter both upflows and downflows. Whereas the upwellings aid the emergence of some segments of the tube, the downflows impede the rise of other parts. Given such an initial configuration, under what circumstances can we expect the tube to emerge coherently without severe distortion by the downflows? In other words, what does it mean to have a "strong" flux tube?

We expect that the flux tube can resist severe distortion by the flow if the field strength satisfies $B_{0} \gtrsim B_{\text {eq }}$, where $B_{\text {eq }}$ is the field strength at which the magnetic energy density and the kinetic energy density of the external flow are in equipartition. The value of the equipartition field strength depends on depth. At the original location of the flux tube $(z=-1.35 \mathrm{Mm})$, the ambient density is $\varrho=4.2 \times 10^{-6} \mathrm{~g} \mathrm{~cm}^{-3}$. The typical vertical velocity of downflowing material at this depth is $4-8 \mathrm{~km} \mathrm{~s}^{-1}$. For this range of velocities, the corresponding range of equipartition field strengths is $B_{\text {eq }}=2900-5800 \mathrm{G}$. Taking the typical values at the surface $\left(\varrho=2.6 \times 10^{-7} \mathrm{~g} \mathrm{~cm}^{-3}, v=2-4 \mathrm{~km} \mathrm{~s}^{-1}\right)$, the 
Table 1. Initial properties of the individual magnetic flux tubes and emerged flux of the simulation runs. The columns give (from left to right) the label of the run, the magnitude of the longitudinal field at the tube axis $\left(B_{0}\right)$, the twist parameter $(\lambda)$, the total longitudinal flux $\left(\Phi_{0}\right)$, the maximum unsigned magnetic flux $\left(\Phi_{\max }^{z=0}\right)$ crossing the $z=0$ plane (i.e. base of the photosphere) within a time period of 20 min after the start of the simulation, and the percentage of tracer fluid elements in the initial flux tube that manage to rise to the photosphere $(z=0)$ within the same time period. In all cases, the characteristic radius of the initial flux tube is $R_{0}=200 \mathrm{~km}$.

\begin{tabular}{cccccc}
\hline \hline Label & $B_{0}(\mathrm{G})$ & $\lambda$ & $\Phi_{0}\left(10^{19} \mathrm{Mx}\right)$ & $\Phi_{\max }^{z=0}\left(10^{19} \mathrm{Mx}\right)$ & Emergence fraction $(\%)$ \\
\hline U1 & 8500 & 0.5 & 1 & 7.7 & 67 \\
U2 & 8500 & 0.25 & 1 & 4.7 & 68 \\
U3 & 8500 & 0.1 & 1 & 3.1 & 44 \\
U4 & 8500 & 0 & 1 & 2.6 & 30 \\
U5 & 8500 & 0.75 & 1 & 11 & 62 \\
U6 & 2500 & 0.5 & 0.31 & 0.9 & 12 \\
U7 & 5250 & 0.5 & 0.66 & 3.0 & 39 \\
U8 & 7000 & 0.5 & 0.86 & 5.6 & 49 \\
\hline
\end{tabular}
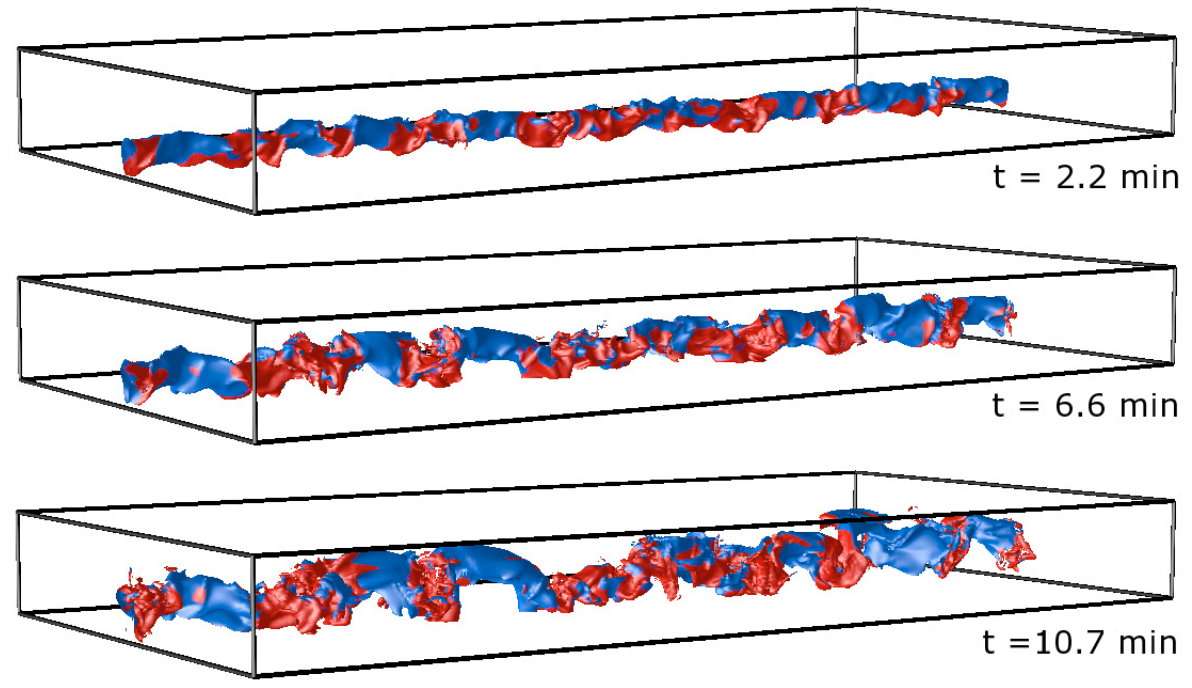

Fig. 1. The passive evolution of a weak magnetic flux tube (run $\mathrm{U} 6, B_{0}=2500 \mathrm{G}$ ) with the convective flow. Shown is a sequence of isosurfaces of $|B|=400 \mathrm{G}$, on which the blue (red) colour-coding indicates upflows (downflows). Segments of the tube embedded in upflows are able to rise and emerge, whereas segments aligned with downflows are kept submerged. equipartition field strength there has much lower values of $B_{\text {eq }}=$ 450-700 G.

Consideration of the force balance on the tube allows us to determine a related, but more stringent criterion. Suppose that the flux tube has uniform magnetic field strength, $B_{0}$, and uniform density deficit, $\Delta \varrho$, with respect to the surroundings. The buoyancy force experienced by the flux tube overcomes the drag force exerted by a downflow of speed $v$ given that (see, for example, Parker 1979; Moreno-Insertis 1983; Schüssler 1984; Schüssler 1987; Fan et al. 2003)

$B_{0} \gtrsim\left(\frac{2 C_{\mathrm{D}} \gamma_{1}}{\pi}\right)^{1 / 2}\left(\frac{H_{p}}{R_{0}}\right)^{1 / 2} B_{\mathrm{eq}}$,

where $H_{p}$ is the local pressure scale height, $C_{\mathrm{D}}$ the drag coefficient (of order unity) and $\gamma_{1}$ the first adiabatic exponent. The factor $2 C_{\mathrm{D}} \gamma_{1} / \pi$ is of order unity. The radius is important because the drag force (per unit length) on the flux tube is proportional to the tube radius while the buoyancy force (also per unit length) is proportional to the square of the tube radius and to the square of the field strength. This means that, for a given field strength, a thick tube tends to be dominated by buoyancy while a sufficiently thin tube is passively dragged by the surrounding flows. For example, we see from the criterion given by Eq. (8) that a tube with a radius $R_{0}=0.25 H_{p}$ must have a field strength of at least $2 B_{\text {eq }}$ in order to have sufficient buoyancy to rise against the drag of the downflows. Since the downflows are localized, the magnetic curvature of a deformed flux tube also acts against the drag force. Like the buoyancy force, the curvature force is also proportional to the square of the magnetic field strength.

Table 1 gives on overview of the various simulation runs carried out. We start the discussion of the results with the "weak" flux tube considered in run U6. The initial field strength at the tube axis is $B_{0}=2500 \mathrm{G}$, corresponding to $\beta=8 \pi p / B^{2} \simeq 22$ there. The characteristic radius of the tube is $R_{0}=200 \mathrm{~km} \simeq$ $0.4 H_{p}$. The tube is initially twisted, with $\lambda=0.5$. The longitudinal magnetic flux threading a cross-section of the tube is $3.1 \times 10^{18} \mathrm{Mx}$. Criterion (8) tells us that the tube must have at least a field strength of $\left(H_{p} / R_{0}\right)^{1 / 2} B_{\text {eq }}=4600-9200 \mathrm{G}$ in order to rise against the downflows. Since the tube has only a central field strength of $B_{0}=2500 \mathrm{G}$ (the average field strength over its cross-section is even smaller), we expect the evolution of the flux tube to be dominated by the convective flows. Figure 1 shows a time sequence of $3 \mathrm{D}$ isosurfaces of $|B|=400 \mathrm{G}$. In all three panels, the blue (red) colour-coding indicates that the vertical velocity at the isosurface is upwards (downwards). Already at 2.2 min after the start of the simulation, the flux tube has been significantly distorted by the convective flows. In the subsequent snapshots, the upflows have advected segments of the tube upwards, whereas the downflows have pinned down other segments below the surface. The shape of the flux tube has developed to a form resembling a sea serpent. The behaviour of the flux tube in the presence of asymmetrical up- and down-flows in compressible convection is reminiscent of the turbulent pumping mechanism studied by Tobias et al. (2001). 

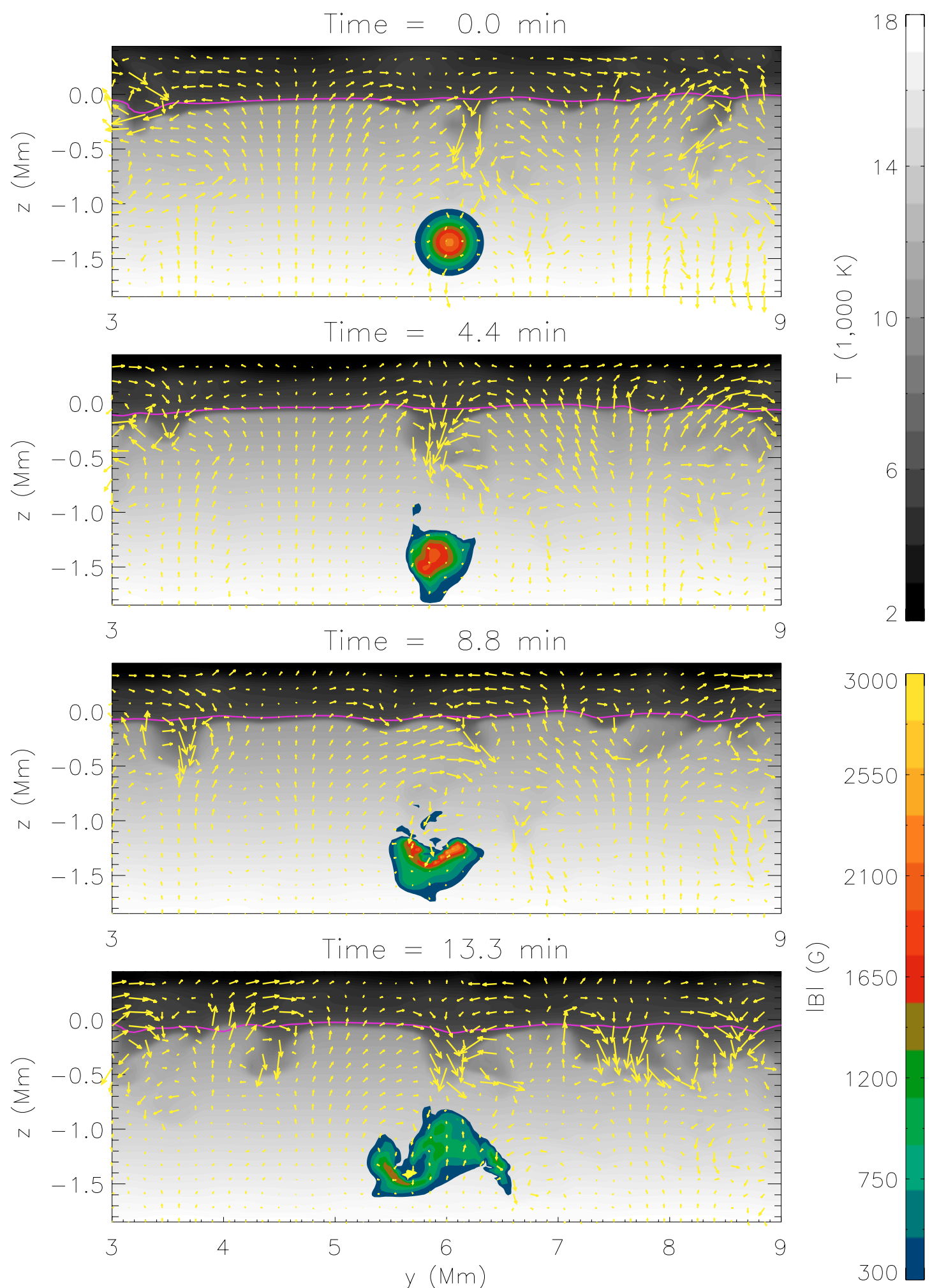

Fig. 2. Time sequence of a vertical cross-section of the simulation domain at $x=12 \mathrm{Mm}$ (perpendicular to the initial tube) for run U6. The tube segment is passively advected by the convecting flow. The greyscale indicates the temperature distribution and the color-coding indicates the distribution of the absolute field strength, $|B|$. The arrows indicate the projection of velocity vectors on the vertical cross-section. The purple line shows the level $\tau_{5000}=1$.

Figure 2 gives another illustration of how the flux tube in run U6 evolves passively with the convecting flow. The figure shows a sequence of snapshots of a vertical cross-section of the simulation domain at $x=12 \mathrm{Mm}$ (perpendicular to the axis of the initial flux tube). The grey scale indicates the temperature distribution while the colour-coding represents the absolute field strength, $|B|$. The arrows indicate the components of the velocity field in the $y z$ plane at $x=12 \mathrm{Mm}$. The purple line running near $z=0$ shows the level of optical depth unity in the continuum at a wavelength of $5000 \AA\left(\tau_{5000}=1\right)$. At $t=0$ min, this particular 

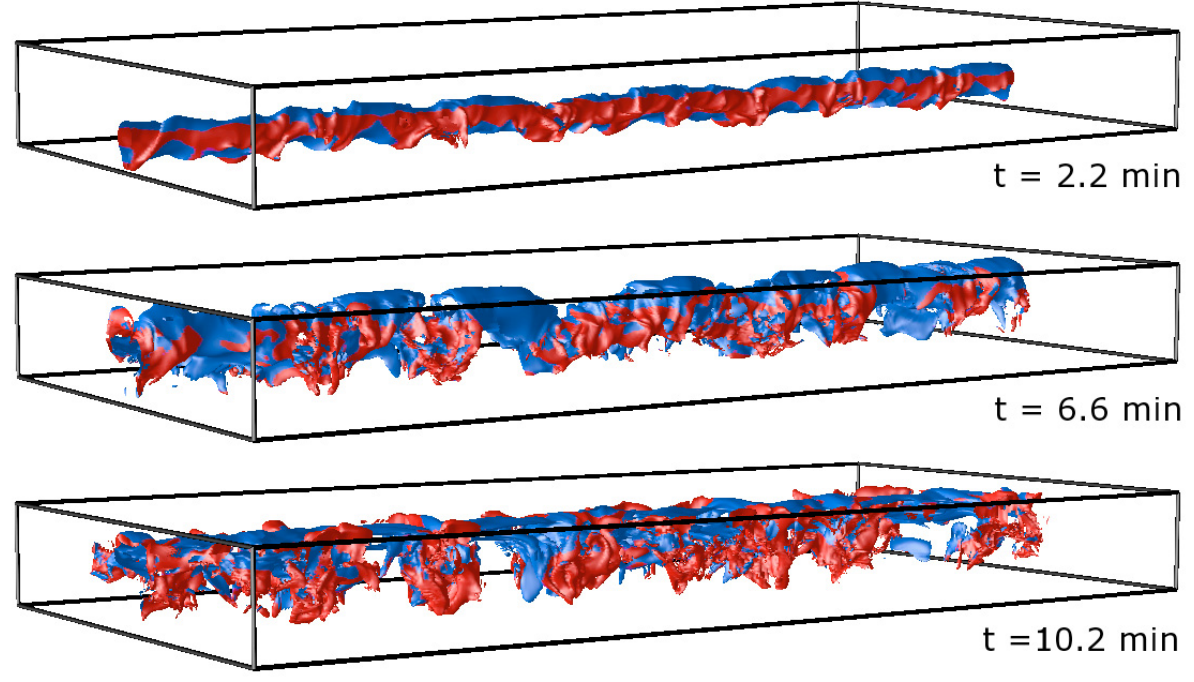

Fig. 3. Same as Fig. 1 but for a flux tube with higher initial field strength (run U1, $B_{0}=$ $8500 \mathrm{G})$. The isosurfaces here correspond to $|B|=700 \mathrm{G}$. In this case, the convective flows do not completely control the dynamics of the flux tube. segment of the tube is aligned with a downflow. In the following snapshot at $t=4.4 \mathrm{~min}$, we find that this segment of the tube has been displaced downwards. In the final two snapshots ( $t=8.8 \mathrm{~min}$ and $t=13.3 \mathrm{~min}$ ), we witness the tube segment being severely deformed by the shear in the velocity field at the interface between upflows and downflows. While the central part of the tube segment is pushed downwards, other parts of the tube segment are carried upwards.

Our simulation runs with higher initial field strengths confirm that the ability of the convective flows to dominate the evolution of the tube lessens with increasing initial field strength. In run $\mathrm{U} 1$, the initial field strength on the axis of the tube is $B_{0}=8500 \mathrm{G}$, which is within the range of $B_{\text {eq }}$ for the original depth of the tube and corresponds to a $\beta \simeq 1$. The total longitudinal magnetic flux carried by the tube is $10^{19} \mathrm{Mx}$. Figure 3 shows a time sequence of isosurfaces of $|B|=700 \mathrm{G}$ for this run. Again, the colour-coding indicates upwards or downwards vertical velocity. In this case, the flux tube is not so weak that it is simply advected by the convective flow. Nor is it sufficiently strong to be unaffected by the flows. The behaviour of the tube is in an intermediate regime between these two extremes. On the one hand, the convective flow is able to deform the tube. On the other hand, the bulk of the tube finally does overcome the impeding downflows and emerges at the surface.

Figure 4 helps emphasize the previous point. At $t=0 \mathrm{~min}$, the tube segment shown is aligned with a downflow. In run U6, this downflow advects the tube downwards. The situation in run $\mathrm{U} 1$ is very different. While the downflow is able to divert the tube slightly towards the right, it is unable to keep the tube beneath the surface. Instead, the tube segment is sufficiently buoyant to overcome the drag by the downflow and finally emerge.

\subsection{Dependence of emerged flux on initial field strength and twist}

\subsubsection{Unsigned flux at the photosphere}

The "fraction" of the original flux tube which manages to emerge at the photosphere can be quantified in different ways. One measure is the maximum value reached in a given time interval by the total unsigned flux through the plane $z=0\left(\Phi_{\max }^{z=0}\right)$. For a time interval of $20 \mathrm{~min}$, this quantity is indicated in the fifth column of Table 1. Since a flux tube emerges as an undulated structure, the emergence actually consists of a collection of small-scale emergence events, each with a typical length-scale of about $1 \mathrm{Mm}$.
This means that the unsigned vertical flux at the photosphere can be several times that of the initial longitudinal flux of the tube $\left(\Phi_{0}\right)$. We identify two clear trends. Firstly, $\Phi_{\max }^{z=0}$ increases with increasing $B_{0}$ for constant $\lambda$. This simply reflects the fact that a magnetic flux tube with higher $B_{0}$ contains more flux (for the same tube radius) and is more buoyant. Secondly, $\Phi_{\max }^{z=0}$ increases with $\lambda$. This is due to the fact that the component of the magnetic field transverse to the original tube axis contributes to the unsigned flux at $z=0$.

\subsubsection{Emergence of tracer fluid elements}

Another measure of the fraction of the tube that emerges can be obtained by considering selected (Lagrangian) fluid elements as tracers of the evolution. To this end, we followed the trajectories of 250 such tracer fluid elements (referred to simply as "tracers" in what follows). The first 50 of them were located equidistantly along the axis of the initial flux tube. The remaining tracers were selected similarly to lie along four lines parallel to the tube axis, each line at a distance of $50 \mathrm{~km}$ from the axis (one line lying above the axis, one lying below, and the other two lines to the left and right of the axis). We determined the fraction of tracers that manage to reach the photosphere $(z \geq 0)$ within the time period $0 \leq t \leq 20 \mathrm{~min}$. The numerical values of the results indicated in the last column of Table 1 and plotted in Fig. 5. For flux tubes with the same initial twist $\lambda$, the percentage systematically increases with the initial field strength, $B_{0}$. As a reference case, we performed a non-magnetic run (i.e. $B_{0}=0$ ) and found that $7 \%$ of the fluid elements emerged within $20 \mathrm{~min}$. In the case of a weak magnetic flux tube (run U6, $B_{0}=2500 \mathrm{G}$ ), $12 \%$ of the tracers emerged, while for a strong tube (run $\mathrm{U} 1, B_{0}=8500 \mathrm{G}$ ), $67 \%$ of the tracers managed to emerge. This trend is due to the fact that the buoyancy of a flux tube scales with $B^{2}$.

The dependence of the percentage of emerged tracers on the twist parameter $\lambda$ is not so straightforward. We see that, at a given magnetic field strength of $B_{0}=8500 \mathrm{G}$, the percentage increases from $30 \%$ to $68 \%$ in the range $\lambda=0-0.25$. This trend is compatible with results from earlier idealized simulations of rising magnetic tubes. These studies found that an untwisted tube, after rising a distance of a few times its diameter, will split into counter-rotating vortex rolls which separate horizontally from each other (Schüssler 1977; Longcope et al. 1996). The fragments of the individual tubes have smaller radii, less flux and are less buoyant. As a result, they are more easily 

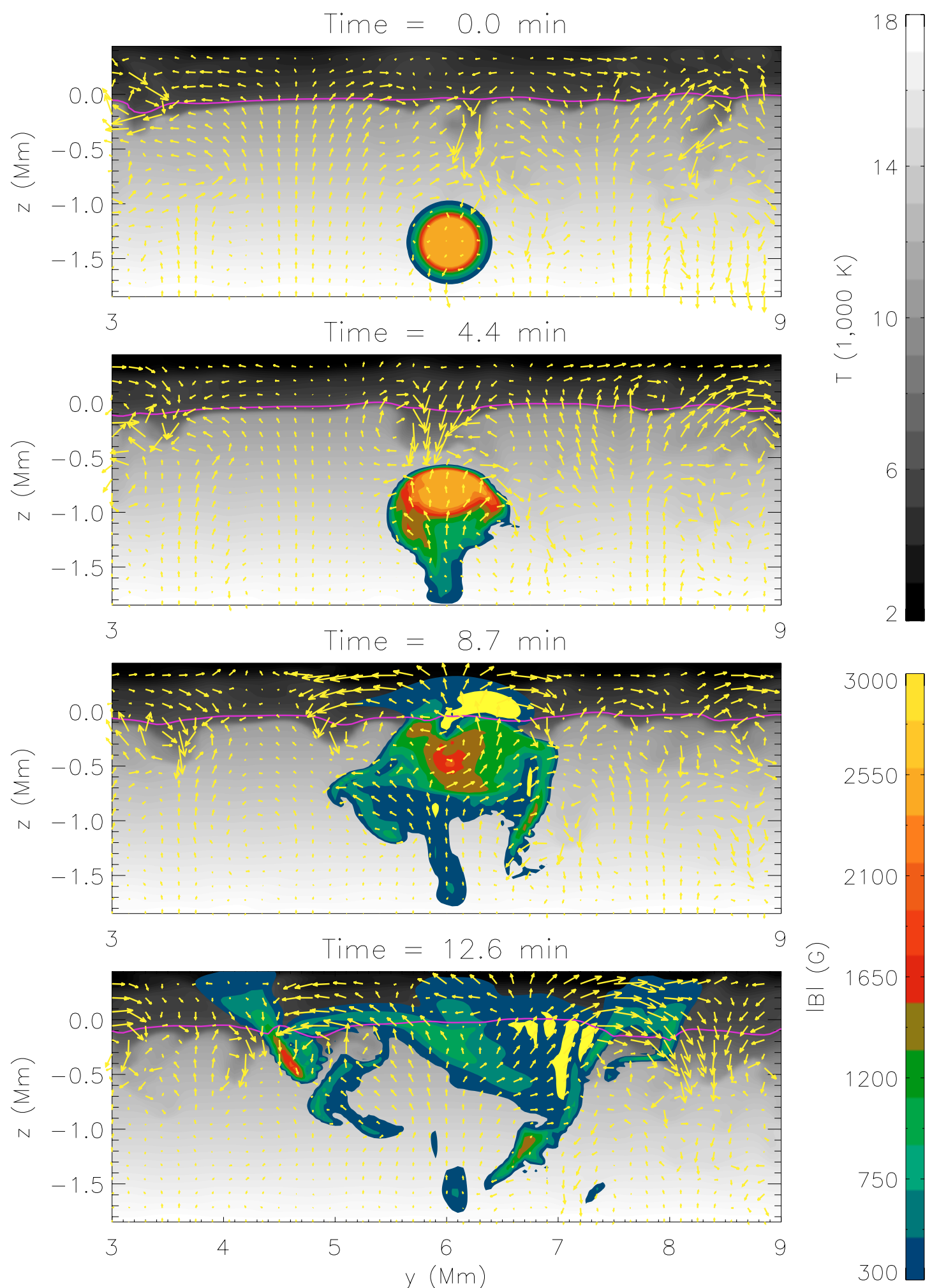

Fig. 4. Same as Fig. 2, but for run U1 $\left(B_{0}=8500 \mathrm{G}\right)$. In this run, the flux tube is sufficiently buoyant and the magnetic field sufficiently strong to rise against the drag of the downflow (see snapshot at $t=0 \mathrm{~min}$ ). The expansion of the rising magnetic complex drives strong horizontal flows away from the emergence site.

disrupted by the external flow. Simulations of twisted magnetic tubes show that the magnetic tension of the transverse field tends to keep the tube coherent (Moreno-Insertis \& Emonet 1996; Fan et al. 1998; Emonet \& Moreno-Insertis 1998; Cheung et al. 2006). This is why the percentage of emerged tracers increases from $\lambda=0$ to $\lambda=0.25$. For $\lambda>0.25$, however, the percentage drops again. This reversal in the trend is not in contradiction with the previous explanation. The complicating factor here is the relationship between the twist of a tube and its buoyancy. For the initial conditions we have chosen (i.e. the divergence of the total stress tensor at $t=0$ closely resembling that of the non-magnetic domain), the density deficit at the core of the tube scales as $\Delta \varrho /\langle\varrho\rangle \sim 1 /\left(1+\lambda^{2} / 2\right)$. Consequently, for a given initial field strength, the density deficit in the core of the tube actually 


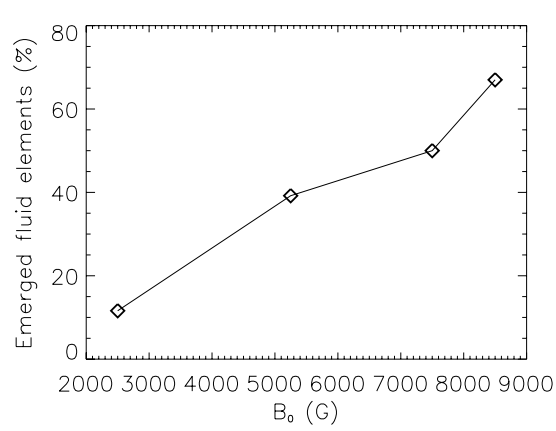

(a) Dependence on $B_{0}$



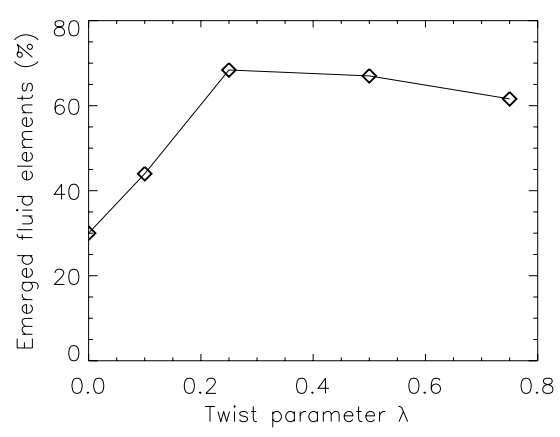

(b) Dependence on twist parameter $\lambda$

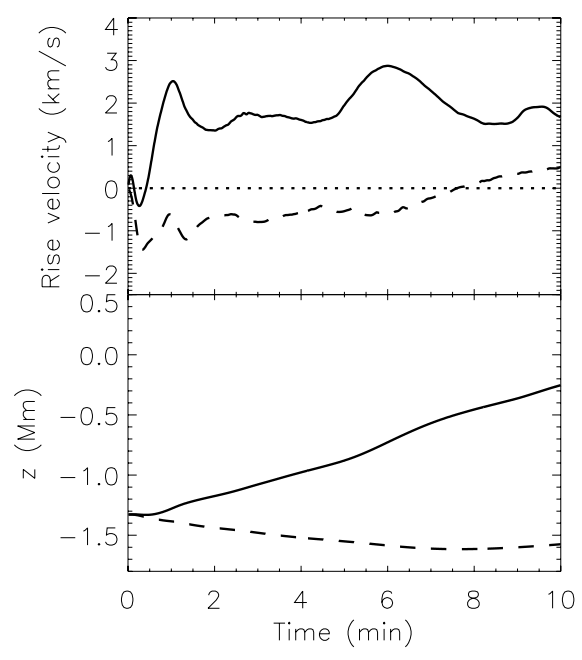

Fig. 5. Percentage of Lagrangian fluid elements initially embedded in the magnetic flux tube that reach the base of the photosphere $(z=0)$ within the time interval $0 \leq t \leq 20 \mathrm{~min}$. decreases with increasing $\lambda$. Hence, although the flux tube remains more coherent with increasing $\lambda$, it is also somewhat less buoyant than the untwisted case.

\subsection{Trajectories of tracer fluid elements}

Figure 6 shows the vertical velocity and height of two tracers initially located at the axis of the magnetic flux tube. Solid and dashed lines correspond to results from run U1 and U6, respectively. The left panel corresponds to a tracer representing a segment of the initial flux tube that was embedded in a convective upflow. While the tracers in both runs accelerate and rise towards the surface, the tracer in run U1 (solid line) experiences larger acceleration and reaches higher rise speeds $\left(v_{y} \approx 2-3 \mathrm{~km} \mathrm{~s}^{-1}\right)$ than the tracer in run U6 $\left(v_{y} \approx 1 \mathrm{~km} \mathrm{~s}^{-1}\right)$. This difference can be attributed to the higher buoyancy of the flux tube in run U1. The right panel of Fig. 6 shows the corresponding results for a tracer in a tube segment initially located in a downdraft. Here, the tracer in run U6 is forced by the downdraft to descend by about $300 \mathrm{~km}$ before it gets caught up in a neighbouring upflow. The tracer in run U1 is also initially pushed downwards by the convective downdraft, but the flux tube in this case is sufficiently buoyant to counteract the downflow.

Figure 7 shows the time evolution of some further properties of the tracer initially embedded in an upflow (Fig. 6, left panel): plasma $\beta$ (top panel), $|B| / \varrho$ (middle), and $Q_{\mathrm{rad}} / \varrho$, the radiative heating rate per unit mass. Until $t \simeq 8 \mathrm{~min}$, the tracer fluid element rises almost adiabatically $\left(Q_{\mathrm{rad}} \approx 0\right)$. During this time, as the fluid rises and expands, the value of $\beta$ increases from an initial value of 1 to a maximum value of 7 . Thereafter, at about $t=8.5 \mathrm{~min}$, the fluid element reaches the transparent photosphere and radiates intensely. The corresponding loss of internal energy corresponds to a decrease of the local gas pressure, leading to a compression of the fluid element and an abrupt decrease of $\beta$ to a value of 3 . Thereafter, the Lagrangian tracer continues to rise through the photosphere (eventually escaping through the top boundary of the domain). As it enters the more tenuous layers of the photosphere, it expands. Despite this, the plasma- $\beta$ hovers for a few minutes at the level of $\beta \approx 3$ and then decreases even further to a value approaching unity. The time profile of the ratio $|B| / \varrho$ (middle panel of Fig. 7) provides a hint as to why this happens. We see that after the fluid has entered the photosphere, $|B| / \varrho$ begins to increase. By virtue of Walen's Equation (derived by combining the equation of continuity and the ideal induction equation, thus ignoring magnetic diffusion),

$\frac{D}{D t}\left(\frac{\boldsymbol{B}}{\varrho}\right)=\frac{1}{\varrho}(\boldsymbol{B} \cdot \nabla) \boldsymbol{v}$

we see that $|B| / \varrho$ increases in the case of stretching of fluid along field lines and decreases in the case of de-stretching.

We can compare the evolution of the tracer (which was initially embedded at the tube axis) with what would result from the simple model of a horizontal thin flux tube rising adiabatically in a stratified, static atmosphere. The result is shown by the dashed curves in Fig. 7. The assumption that there are no variations along the direction of the tube axis means that $|B| / \varrho$ is constant (flat dashed line in the middle panel). Assuming adiabatic rise and instantaneous total pressure balance with the (prescribed) surroundings of the thin tube, we can determine the evolution of the properties of the thin flux tube as functions of its initial state and its vertical displacement (Cheung et al. 2006). 


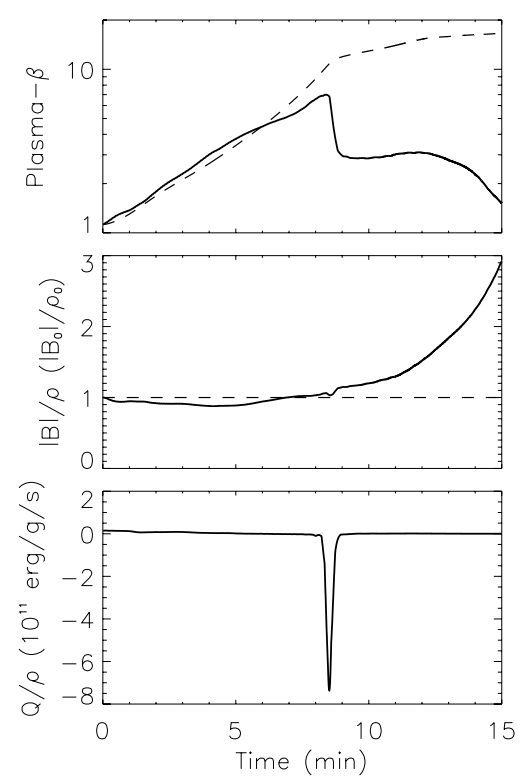

Fig. 7. Temporal evolution of plasma $\beta$, ratio $|B| / \varrho$, and specific radiative heating, $Q_{\mathrm{rad}} / \varrho$, for a tracer fluid element initially located at the axis of the tube segment embedded on a convective upflow (solid curves). The dashed curves indicates values corresponding to a thin flux tube model assuming adiabatic motion and no variation along the tube axis. As long as there is negligible radiative heating and draining of mass at the position of the tracer, there is a good match between the thin-tube model and the actual profiles.

Between $t=0$ and $t=8 \mathrm{~min}$, there is very good agreement between the actual and thin-tube model profiles, resulting from the quasi-adiabatic motion and negligible amount of mass drainage or accumulation along the field lines. Once the Lagrangian tracer reaches the surface and loses entropy, the profiles of $\beta$ diverge. Whereas the actual value of $\beta$ decreases sharply (due to cooling) and then more gradually (due to mass drainage), the thin-tube model predicts that $\beta$ continues to rise smoothly as the fluid continues to rise in the photosphere. The actual and model profiles of $|B| / \varrho$ also diverge. Since our thin-tube model does not take into account variations along the tube, $|B| / \varrho$ remains constant in the model profile. In the actual profile, $|B| / \varrho$ increases after the fluid elements has emerged. This is ultimately a result of radiative cooling, which makes the emerged fluid denser than the fluid immediately below, driving the development of a downflow. This accelerating material in the developing downflow stretches the fluid along the field line and increases $|B| / \varrho$.

\section{Observational signatures}

In the following sections, we compare diagnostics derived from the simulation results with observations of flux emergence.

\section{1. "Quiescent" flux emergence}

The tube in run U6 evolves passively with the convective flow. Consequently, the emergence of magnetic flux does not lead to a severe disturbance in the appearance of the granulation in white light intensity images. For this reason, we refer to these flux emergence events as quiescent.

Figure 8 shows a sequence of continuum intensity and magnetic field maps representing the flux emergence event in run U6. The magnetic field is evaluated at $\tau_{5000}=0.1$ (optical depth of 0.1 for the visible continuum at $5000 \AA$ ). We chose to sample from this optical depth because the maxima of the contribution functions of many photospheric lines commonly used to probe surface magnetic fields (e.g. Fe I 6301, Fe I 6302) are roughly located at this depth. As such, we can expect that the information carried by the line to be representative of the physical quantities in this layer of the atmosphere. In the left panels of Fig. 8, the overlaid colored contours indicate the vertical component of the magnetic field $\left(B_{z}\right)$.

The flux tube is initially aligned in the $x$-direction at $y=$ $6 \mathrm{Mm}$. At $t=12.4 \mathrm{~min}$, we see the first signs of flux emergence at the surface. The magnetic field emerges within the interior of granules, being predominantly horizontal near the granular centre. Towards the edge of the granule, the magnetic field becomes more vertical, indicating that the magnetic field threading a granule is forced to emerge in an arched configuration. One example is the bipolar region emerging in the granule centered at $[x, y]=[4,7] \mathrm{Mm}$ (see snapshot at $t=12.4 \mathrm{~min}$ ). At this particular instant, the flux contained in each polarity of this small bipole is $8 \times 10^{17} \mathrm{Mx}$. The diverging horizontal flow of the granule flow has the effect of expelling the emerged flux into the network of intergranular downflow lanes within a typical granulation timescale on the order of 5 min (Bray et al. 1984; Title et al. 1989). This finding is in accordance with earlier simulations of magneto-convection in an idealized setup (Weiss 1966; Proctor \& Weiss 1982; Hurlburt \& Toomre 1988) as well as more recent, realistic simulations of magneto-convection in the near-surface layers of the solar convection zone (Steiner et al. 1998; Bercik 2002; Bercik et al. 2003; Vögler et al. 2005; Stein \& Nordlund 2006). At $t=17.7 \mathrm{~min}$ (about $5 \mathrm{~min}$ after the first snapshot), the distribution of the vertical field $B_{z}$ already shows that the regions of strongest field are in the intergranular lanes. However, the surface field strength at $\tau_{5000}=0.1$ rarely exceeds the equipartition value of $450 \mathrm{G}$. In comparison, the granule interiors have significantly weaker fields. The next snapshot at $t=22.1$ min illustrates the further transport and dispersion of the emerged flux in the intergranular network.

How do our simulation results compare with observations? De Pontieu (2002) has obtained high cadence and high resolution observations of an emerging flux region (EFR). Within a time series of $3.5 \mathrm{~h}$ duration, he identified seven individual small-scale emergence events. His two key findings relevant for this discussion are: (a) magnetic concentrations emerge in the interior of granules; and (b) within 10-15 min from their initial appearance, the flux concentrations are quickly dispersed by the granular flow. He estimates the average flux density of the emerging magnetic concentrations to be about $200 \pm$ $30 \mathrm{Mx} \mathrm{cm}$. . If this value were to correspond to the actual magnetic field strength, the emerging fields would have subequipartition strength $\left(B_{\text {eq }} \approx 450 \mathrm{G}\right.$ ), a finding consistent with his results (a) and (b). De Pontieu also gives estimates for the average flux of the emerging concentrations in the range $(9 \pm 4) \times$ $10^{17} \mathrm{Mx}$. This is the same order of magnitude as what we find for individual polarities of emerging flux concentrations in run U6.

De Pontieu suggested that the small-scale emergence events he has detected may be related to the so-called horizontal internetwork fields (HIFs, Lites et al. 1996), which are predominantly horizontal magnetic structures with angular and time scales of $1-2^{\prime \prime}$ and $5 \mathrm{~min}$, respectively. The existence of HIFs are inferred from the distinctive signals in the Stokes $Q$ and $U$ profiles of relatively quiet regions of the solar surface. The strength of these signals indicate that HIFs are generally weak with $|B| \lesssim 600 \mathrm{G}$. HIFs are usually detected in regions where the spectral lines are slightly blueshifted, indicating an association 

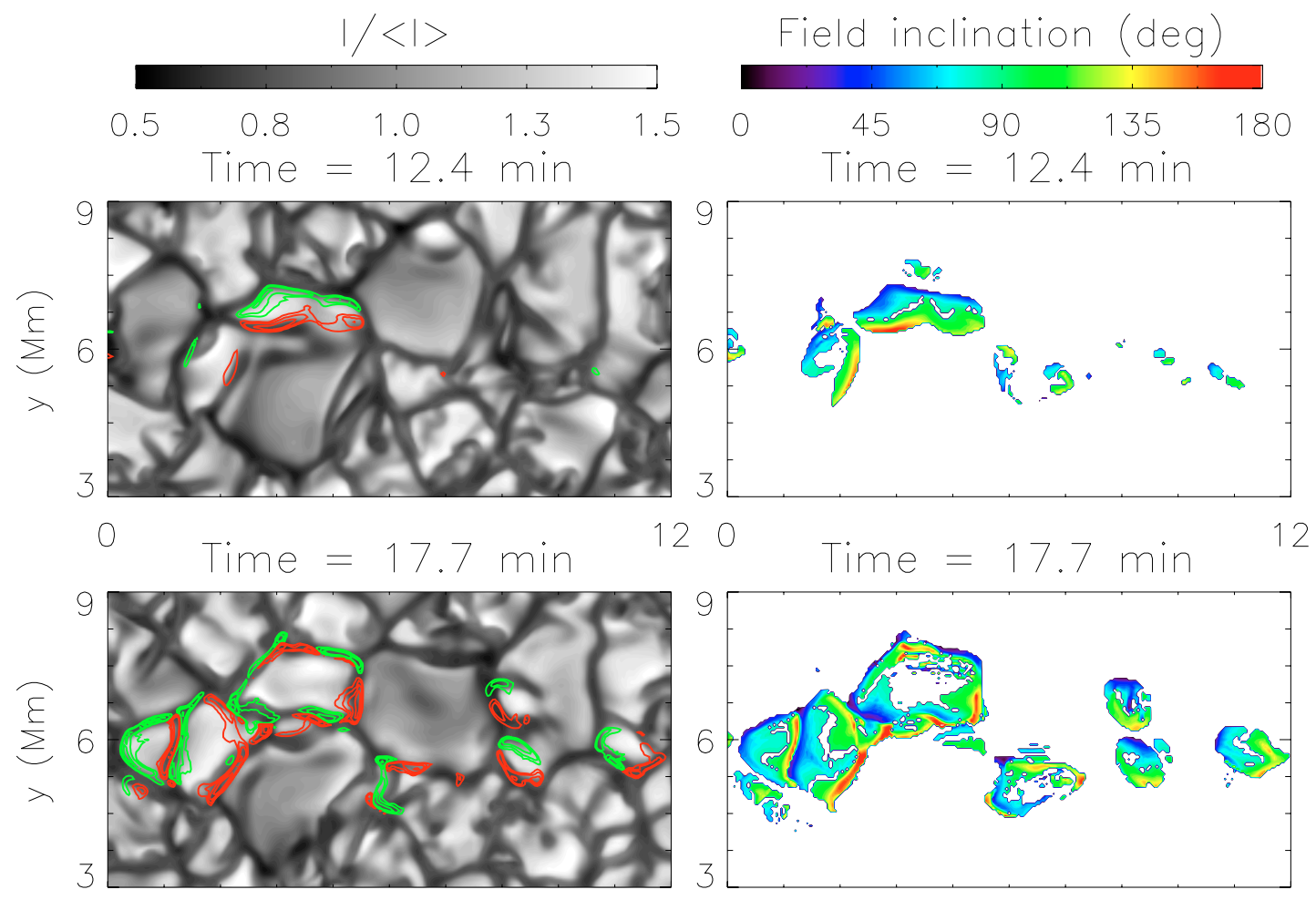

12

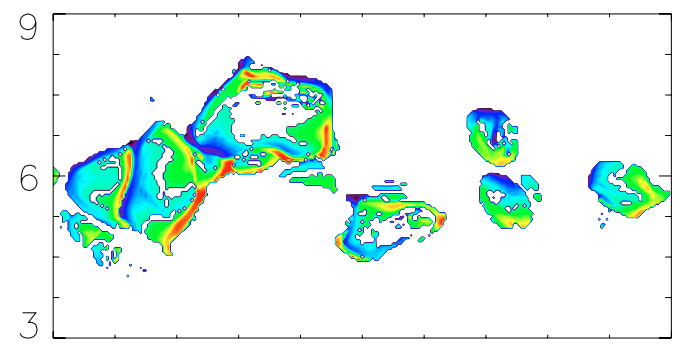

0

12

0

Time $=22.1 \mathrm{~min}$
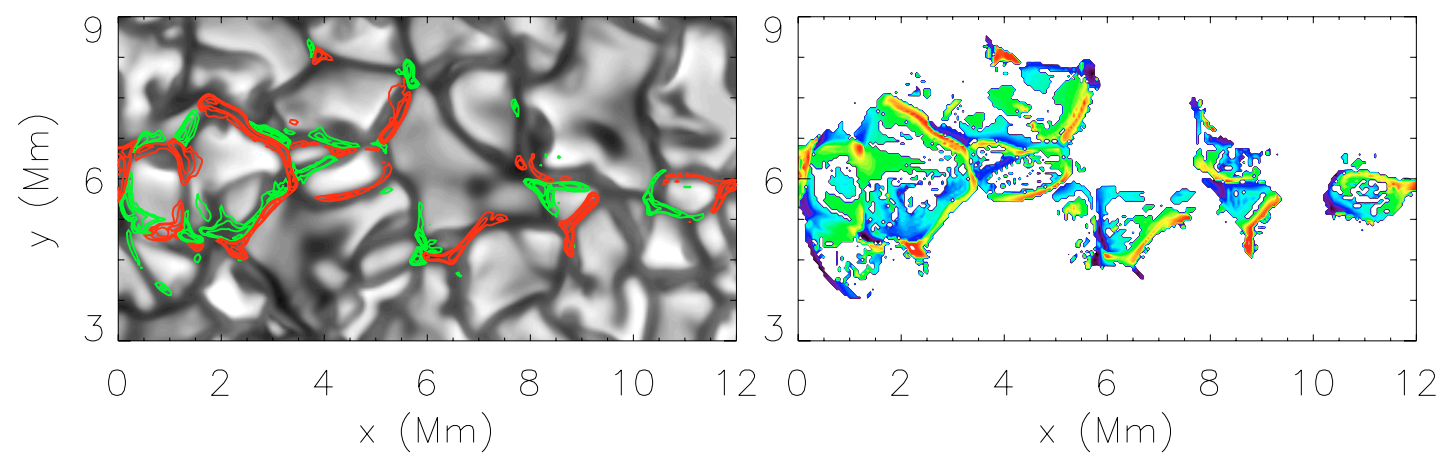

Fig. 8. Emergence of magnetic field into the photosphere in run U6. Left column: time sequence of the vertically emerging continuum intensity at $5000 \AA$ A $I_{5000}$. Right column: the corresponding time sequence of magnetic field inclination evaluated at $\tau_{5000}=0.1$. A field inclination (also called zenith angle) of $0^{\circ}$ and $180^{\circ}$ respectively correspond to vertical magnetic field directed out of and into the $x-y$ plane. In the left panels, the color contours indicate the vertical component of the magnetic field at $\tau_{5000}=0.1$ with the levels $\pm[50,100,200,400]$ G. Green (red) contours indicate magnetic upward (downward) directed field. An accompanying mpeg animation is available.

with upflows. Both Lites et al. and De Pontieu interpret these as signatures of the crests of magnetic loops emerging in granules.

We have analyzed simulation run U6 to check whether this interpretation is consistent with our results. Figure 9 shows joint probability distribution functions (JPDFs) of different surface quantities in the simulation run for times $t=12.4 \mathrm{~min}$ (upper row) and $t=61.1$ min (lower row). The left column shows JPDFs between the zenith angle $\gamma$ of the magnetic field and the absolute field strength, $|B|$, both evaluated at $\tau_{5000}=0.1$. The zenith angle is defined as the angle between $\boldsymbol{B}$ and the vertical direction ( $z$-axis). As such, magnetic field vectors with $\gamma=0^{\circ}$ and $\gamma=180^{\circ}$ correspond to purely vertical magnetic field that point out of and into the horizontal plane, respectively. Purely horizontal fields have $\gamma=90^{\circ}$. The right column shows JPDFs of the zenith angle of $\boldsymbol{B}$ and the vertical fluid velocity (for surface regions with $|B|>0$ ). Flux begins to emerge approximately at $t=12.4 \mathrm{~min}$. The JPDFs at this time indicate the existence of predominantly horizontal field with strengths of up to
$|B| \approx 400 \mathrm{G}$. These horizontal fields mainly have rise velocities of $1-2 \mathrm{~km} \mathrm{~s}^{-1}$, which is typical of granular upflows. This finding is compatible with the scenario proposed by Lites et al. (1996) and by De Pontieu (2002) for the explanation of HIFs.

The snapshot taken at $t=61.1$ min (lower panels in Fig. 9 shows a distinctly different shape for the JPDF between the zenith angle and $|B|$. The pair of horn-like features indicate strong vertical fields of up to $1 \mathrm{kG}$. We will return to this point in the following section. Furthermore, there is a continuous distribution of nearly horizontal fields with strengths of up to $300 \mathrm{G}$. The accompanying JPDF between the zenith angle and $v_{z}$ indicates that the majority of horizontal fields reside within upflow regions (granules). In fact, the JPDF between the zenith angle and $|B|$ at $t=61.1$ min resembles those found in simulations of magneto-convection in the near-surface layers with a net vertical magnetic flux threading the simulation domain (Vögler 2003). This indicates that the abundance of weak horizontal fields in 

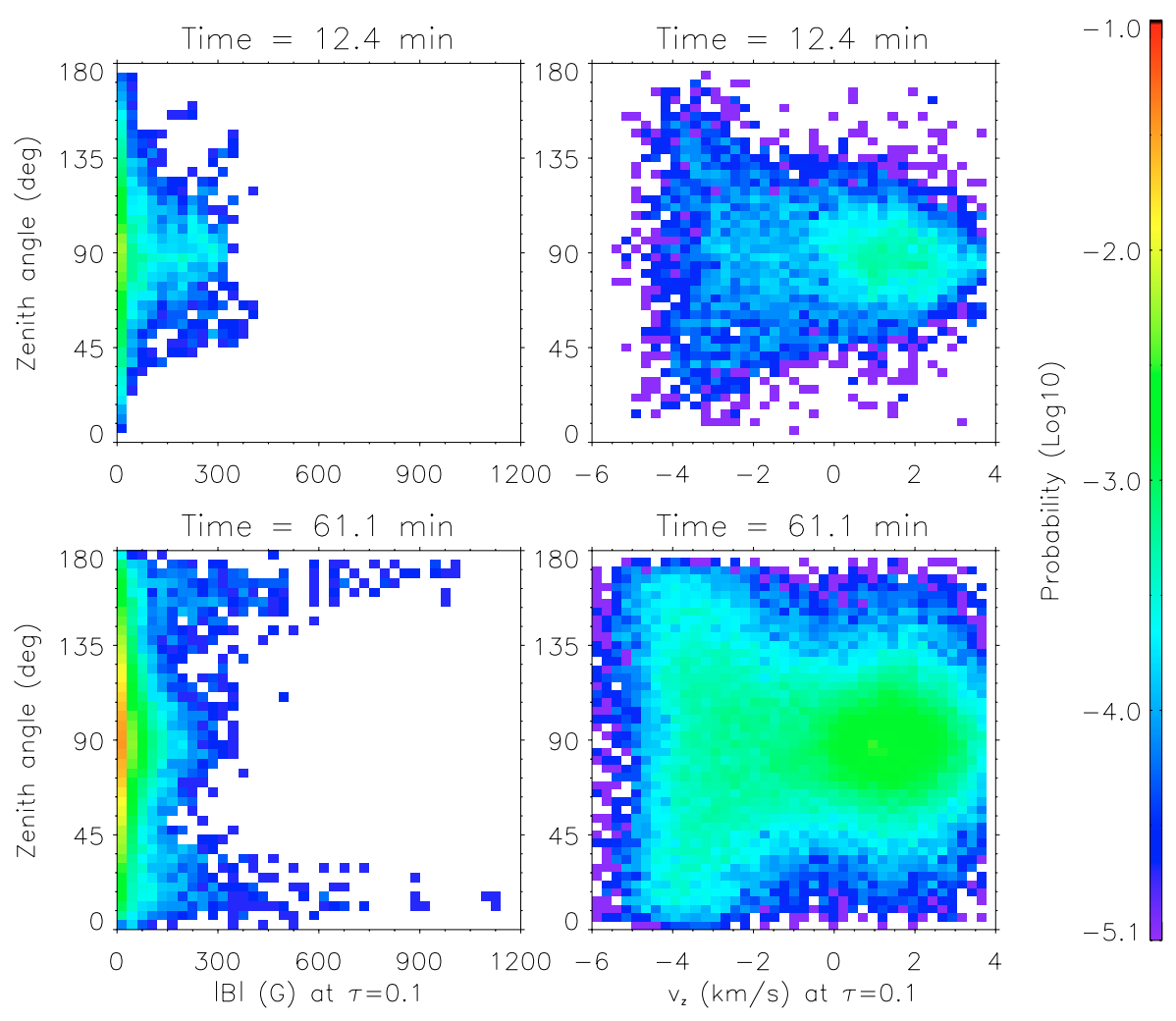

Fig. 9. Left column: joint probability distribution functions (JPDFs) of the zenith angle of the magnetic field vector and the absolute field strength, $|B|$, both at the level $\tau_{5000}=0.1$. Right column: JPDF of zenith angle and vertical fluid velocity. The colour coding indicates the logarithm of the probability. The upper and lower rows, respectively, show the JPDFs at $t=12.4 \mathrm{~min}$ and $t=61.1 \mathrm{~min}$ for run $\mathrm{U} 6$. granule interiors is a general feature of magneto-convection and not necessarily always associated with emerging flux.

\subsubsection{Surface evolution of emerged field: cancellation, coalescence and secondary emergence}

In run U6, the morphology of the emerged field bears little resemblance to the initial horizontal flux tube structure. The arrangement of the surface field after the initial flux emergence (see snapshots at $t=17.7$ and $22.1 \mathrm{~min}$ in Fig. 8) resembles the "salt and pepper" pattern of quiet Sun magnetic fields observed, e.g., by Domínguez Cerdeña et al. (2003), displaying a mixture of positive and negative small-scale flux in the intergranular network. These authors describe instances when flux concentrations of like polarities meet and appear to coalesce (to within the resolution limit). They also show a flux cancellation event due to the encounter of opposite polarities. In our simulation run U6, both these types of events occur as well. In the following, we discuss in detail an instance of a coalescence event between vertical flux concentrations of like-polarity.

Figure 10 shows a time sequence of intensity images in the vicinity of a granule, overplotted with horizontal velocity vectors and contours of the vertical magnetic field strength, both at $\tau_{5000}=0.1$. At time $t=47.9 \mathrm{~min}$, we find a number of magnetic flux concentrations of both polarities. The most prominent feature is centered at $[x, y]=[2.5,9] \mathrm{Mm}$. This feature resides at a downflow vertex of intergranular lanes and has a flux of $1.5 \times 10^{18} \mathrm{Mx}$. The core of the feature has a maximum vertical field strength of $B_{z}=1,200 \mathrm{G}$ (at $\tau_{5000}=0.1$ ). A few minutes later, at $t=52.1 \mathrm{~min}$, we find a small bipole emerging through a granule that is located to the right of the preexisting flux concentration. The bipole is oriented such that the pole closest to the pre-existing flux concentration has the same polarity (positive). The newly emerged flux is subsequently expelled to the adjacent downflow lane, where it coalesces with the pre-existing feature. The resulting larger flux concentration has a net flux of $2.6 \times 10^{18} \mathrm{Mx}$, comparable to the longitudinal flux of the horizontal flux tube at the beginning of the simulation $\left(\Phi_{0}=3.1 \times 10^{18} \mathrm{Mx}\right)$.

This particular example is interesting for a number of reasons. Firstly, the new bipole emerges at a relatively late stage of the simulation, about $40 \mathrm{~min}$ after the initial appearance of flux at the surface (see Fig. 8). Its emergence location is also unusual. In Fig. 8, we see that almost all the flux emerges between $y=4$ and $y=8 \mathrm{Mm}$. The new bipole, however, emerges significantly further away, at $y=9.2 \mathrm{Mm}$. These two points are consistent with the fact that, when a section of a flux tube is passively carried by an upwelling, not all the flux contained in the tube emerges in one single event. The material near the edge of an upwelling may overturn before it reaches the surface. Consequently, the magnetic field threading this material fails to emerge on first attempt. This magnetic field may then continue to travel downwards, or may get caught up in another upwelling and travel upwards again. The emergence of the bipole in Fig. 10 is one such example. The magnetic field associated with this bipole has overturned several times before it eventually reaches the $\tau_{5000}=0.1$ surface. Considering that it had been caught up in different upflows and downflows, it is not surprising that its emergence location is about $1 \mathrm{Mm}$ further afield.

The coalescence event shown in Fig. 10 is interesting for another reason. The pre-existing positive flux concentration residing in a downflow vertex is not as dark as the average intergranular network. After coalescence, the resulting concentration becomes even brighter. To explain this, we make reference to Fig. 11. Let us first consider the top row of this figure, which shows joint probability distribution functions (JPDFs) between the value of $\left|B_{z}\right|$ at a horizontal plane of constant geometrical height $\left\langle z_{0.1}\right\rangle$ and its value evaluated at optical depth $\tau_{5000}=0.1$. The height $\left\langle z_{0.1}\right\rangle$ is defined as the average geometric height of the $\tau_{5000}=0.1$ surface in the absence of magnetic fields. On 


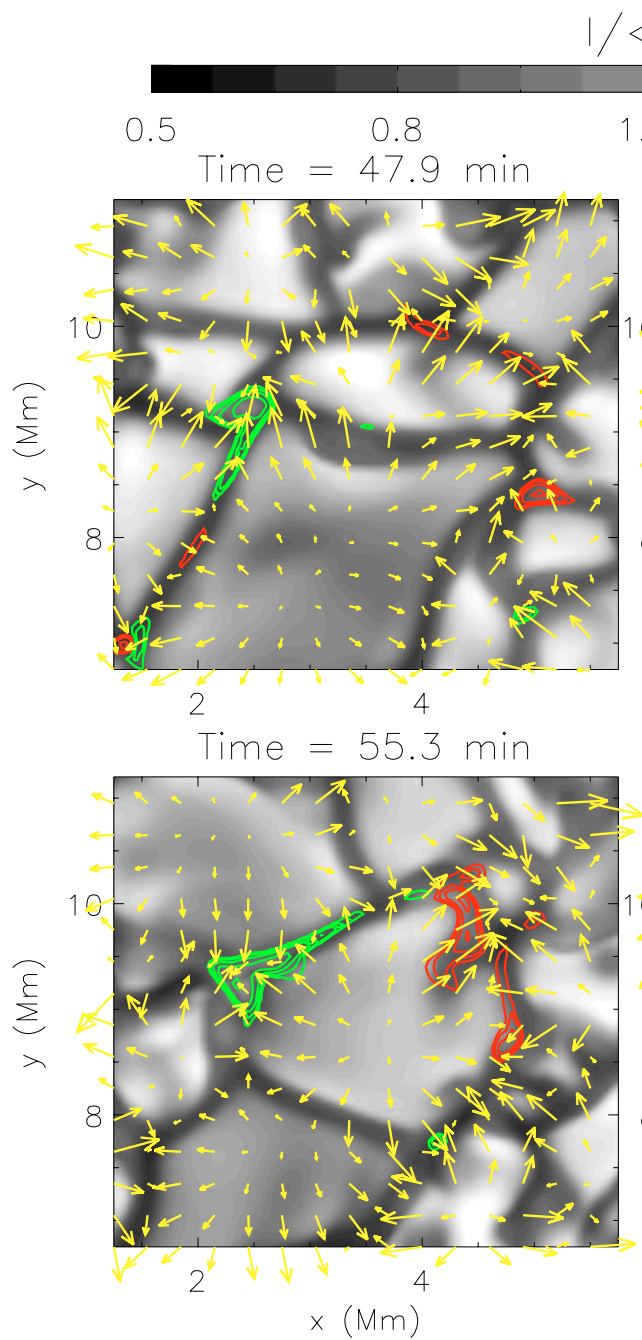

$/<1>$
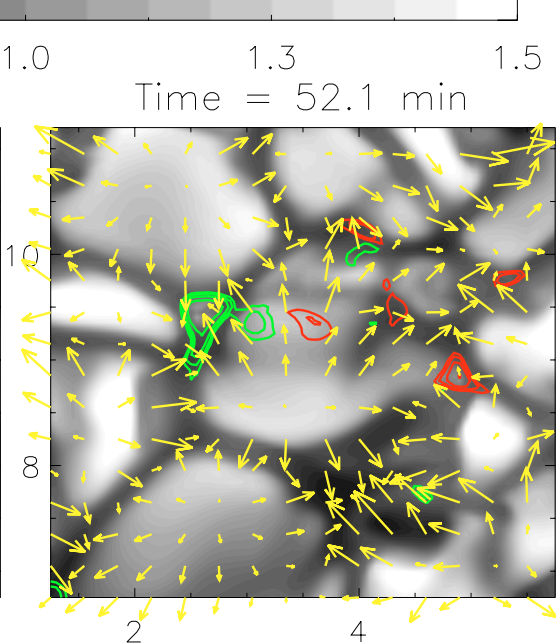

2

Time $=58.3 \mathrm{~min}$

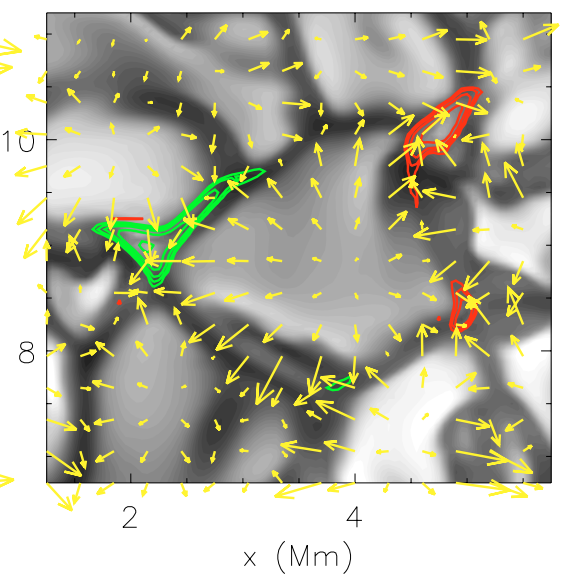

Fig. 10. Coalescence of newly emerged magnetic concentrations with pre-existing surface field in simulation run U6. The grey shading in each panel represents the normalized continuum intensity at $5000 \AA$. The vector overlays indicate the horizontal velocity field at optical depth $\tau_{5000}=0.1$ and the contour overlays indicate $B_{z}$ at the same optical depth at the levels $\pm[100,200,400,800,1600]$ G. Green (red) contours correspond to positive (negative) polarities. At $t=52.1 \mathrm{~min}$, a bipole centered at $[x, y]=[3.5,9.2] \mathrm{Mm}$ is emerging through a granule. Flux expulsion leads to coalescence of the pre-existing positive flux with the positive part of the newly emerged flux. average, the $\tau_{5000}=0.1$ surface lies about $160 \mathrm{~km}$ above the level $\tau_{5000}=1.0$. We first focus on the distribution of field strengths at the horizontal plane $z=\left\langle z_{0.1}\right\rangle$ (i.e. ignore the $y$-axis). A few minutes after the initial appearance of flux at the surface $(t=17.7 \mathrm{~min})$, the vertical field strengths do not exceed $500 \mathrm{G}$. Some time later, at $t=34.6 \mathrm{~min}$, a small fraction of the vertical flux concentrations have reached field strengths of up to $700 \mathrm{G}$, and at $t=61.1 \mathrm{~min}$ we find fields approaching $\left|B_{z}\right|=1 \mathrm{kG}$ at $z=\left\langle z_{0.1}\right\rangle$.

The appearance of stronger magnetic fields several granulation timescales after the initial emergence of flux suggests that convective intensification operates (e.g., Nordlund \& Stein 1990; Grossmann-Doerth et al. 1998; Vögler et al. 2005; Stein $\&$ Nordlund 2006). The magnetic field maps from run U6 show that the maximum value of $\left|B_{z}\right|$ within a vertical magnetic bundle is an increasing function of its total magnetic flux, $\Phi$. In order to identify individual flux bundles, we choose a threshold value, $B_{\text {th }}$. For each discrete region in the magnetic field map with $\left|B_{z}\right| \geq B_{\text {th }}$, we measured the maximum value of $\left|B_{z}\right|$ in the region as well as the amount of flux contained within the region. A scatter plot of these two quantities is shown in Fig. 12. The crosses, diamonds and squares correspond to the snapshots taken at $t=17.7 \mathrm{~min}, t=34.6 \mathrm{~min}$, and $t=61.1 \mathrm{~min}$, respectively, for $B_{\mathrm{th}}=50 \mathrm{G}$. The scatter plot clearly shows that larger flux bundles can support stronger internal field strengths. This trend is not sensitive to the threshold value used. Our finding is compatible with the work of Venkatakrishnan (1986) - who suggested that the radiative heating of flux tubes by their surroundings inhibits the intensification of very small flux tubes and with the observational study by Solanki et al. (1996). We should point out, however, that the various types of diffusion present in the simulation affect the results for the smallest flux concentrations. For instance, a flux bundle with a mean field strength of $100 \mathrm{G}$ and a flux of $10^{16} \mathrm{Mx}$ has a radius of about $50 \mathrm{~km}$, equal to the horizontal grid spacing used in our simulations, so that it is essentially unresolved. Values in the scatter plot in the range $\Phi \lesssim 10^{17} \mathrm{Mx}$ are therefore probably influenced by diffusive effects.

For the snapshot taken at $t=61.1 \mathrm{~min}$, the maximum field strength at $z=\left\langle z_{0.1}\right\rangle$ has reached about $1 \mathrm{kG}$. The corresponding magnetic pressure is almost equal to the horizontally averaged gas pressure at that geometrical level. This indicates that the strongest flux concentrations are largely evacuated, leading to a downward shift of the optical depth scale within the flux concentration ("Wilson depression"). The relation between the displacement, $z_{0.1}-\left\langle z_{0.1}\right\rangle$, and the field strength is shown in the second row of JPDFs in Fig. 11: the stronger the field, the larger is the downward (negative) displacement of the $\tau_{5000}=0.1$ level, which can reach values up to $120 \mathrm{~km}$. This has the consequence that $\left|B_{z}\right|$ at the local level $\tau_{5000}=0.1$ has values of up to $1.5 \mathrm{kG}$, since the field strength increases with geometrical depth (see the top right panel in Fig. 11).

The partial evacuation of vertical flux concentrations leads to another observational signature. From the Eddington-Barbier 


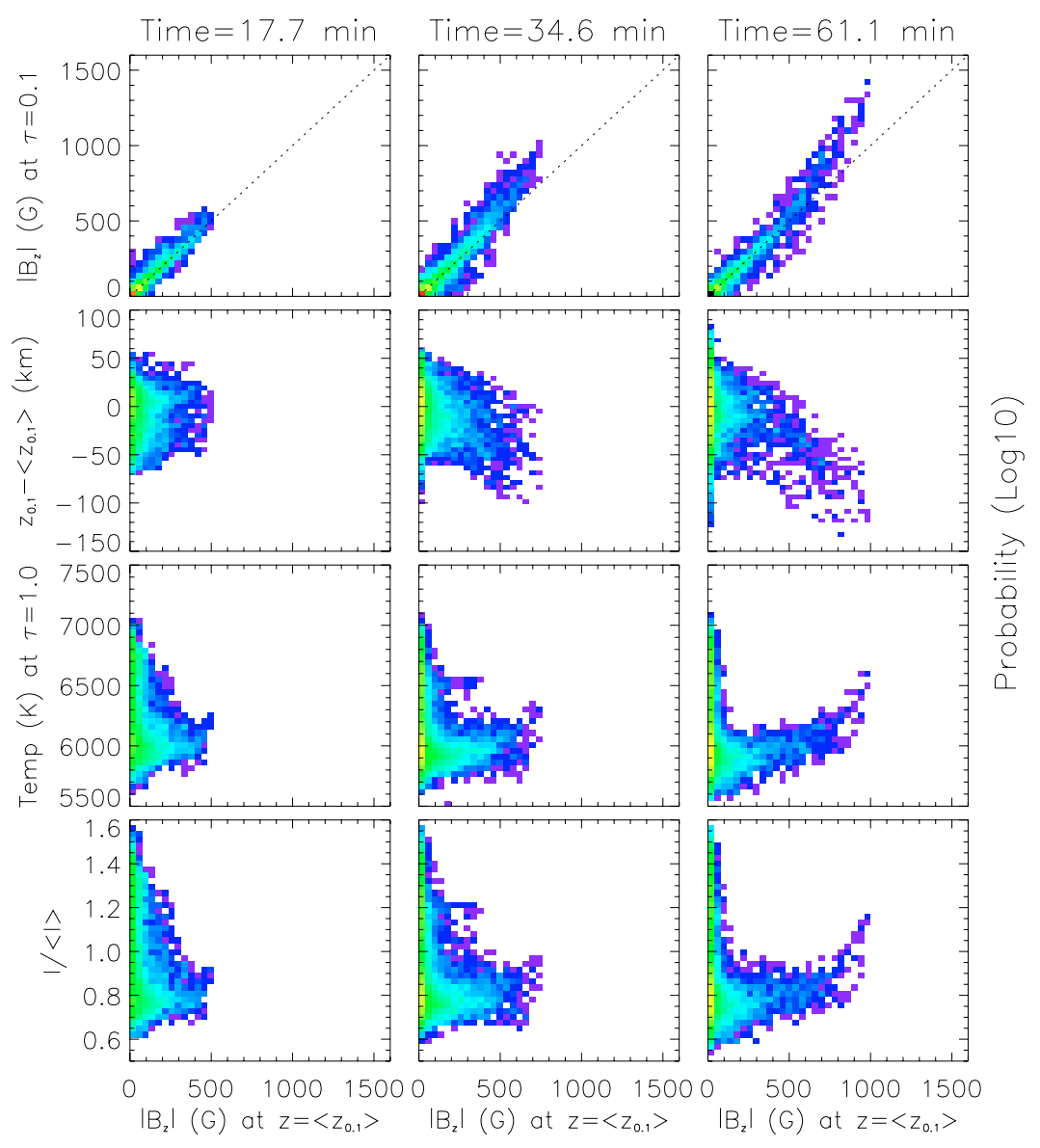



Fig. 11. Joint probability distribution functions (JPDFs) for various quantities in run U6. In all panels, the horizontal axis gives the value of $\left|B_{z}\right|$ at a plane of constant geometrical height, $z=\left\langle z_{0.1}\right\rangle$. The quantities given by the vertical axis are: $\left|B_{z}\right|$ at the local level $\tau_{5000}=$ 0.1 (first row of panels), the local vertical displacement of the $\tau_{5000}=0.1$ surface from the mean geometrical height of the surface corresponding to this optical depth $\left(z_{0.1}-\left\langle z_{0.1}\right\rangle\right.$, second row $)$, the temperature at $\tau_{5000}=1.0$ (third row), and the normalized emergent continuum intensity at $5000 \AA$ (fourth row). The three columns show the JPDFs at three different times. The color coding indicates the logarithm of the probability.



Fig. 12. Scatter plot of the maximum value of $\left|B_{z}\right|$ in individual magnetic flux concentrations versus the amount of vertical flux contained in the flux concentration. The values of $\left|B_{z}\right|$ were evaluated at a plane of constant geometrical height, $z=\left\langle z_{0.1}\right\rangle$. The crosses, diamonds and squares indicate values corresponding to the snapshots taken at $t=17.7 \mathrm{~min}$, $t=34.6 \mathrm{~min}$, and $t=61.1 \mathrm{~min}$, respectively.

relation, we know that the emergent intensity is approximately given by the source function at $\tau=1$. The panels in the third row of Fig. 11 show JPDFs between $\left|B_{z}\right|$ at $z=\left\langle z_{0.1}\right\rangle$ and the temperature at $\tau_{5000}=1.0$. The temperature at optical depth unity is higher in strong field regions, with the result that the brightness of vertical flux tubes increases with $\left|B_{z}\right|$ (see fourth row of Fig. 11). The higher temperature at $\tau_{5000}=1.0$ is due to the lateral radiative heating of the interior of the flux concentration by the surrounding "hot walls" (Spruit 1976; Deinzer et al. 1984; Grossmann-Doerth et al. 1994; Vögler et al. 2005).

\subsection{Emergence of strong magnetic field}

In this section, we discuss the observational signatures of the emerging flux tube with $B_{0}=8500 \mathrm{G}$ (run U1), which is strong enough to resist being passively deformed and advected by the convective flow. Figure 13a gives a time sequence of continuum intensity maps throughout the emergence event (grey scale). The vector overlays indicate the horizontal components of the velocity field at $\tau_{5000}=0.1$. Figure $13 \mathrm{~b}$ shows the corresponding maps of the vector magnetic field.

We have seen that the emergence morphology of the field in run U6 is dictated by the convective motion of the near-surface layers. In run U1 (where the magnetic field is several times stronger), the convection still has an appreciable influence on the emergence morphology. Comparison of the magnetic field and the intensity maps at $t=8.6$ min clearly shows that magnetic flux emerges preferentially within the interior of granules. On the other hand, we find that the emerging magnetic structure also affects the appearance of the local granulation pattern. At $t=8.6 \mathrm{~min}$ (top panel of Fig. 13a), there is an alignment of granules along the direction of the tube axis. These granules are relatively large, each covering an area of up to $9 \mathrm{Mm}^{2}$. The disturbance of the granulation by the emerging magnetic field is most prominent at $t=11.2 \mathrm{~min}$, showing a darkening near the emergence site extending over the full horizontal size of the simulation domain. This darkening is not coherent along its entire length: there are bright patches on either side of the darkening but their shapes are distinctly different from those of normal granules. The expansion of the emerging tube drives horizontal outflows away from the emergence site, which are visible in the vector plots in the middle panel of Fig. 13a. The magnetic field 

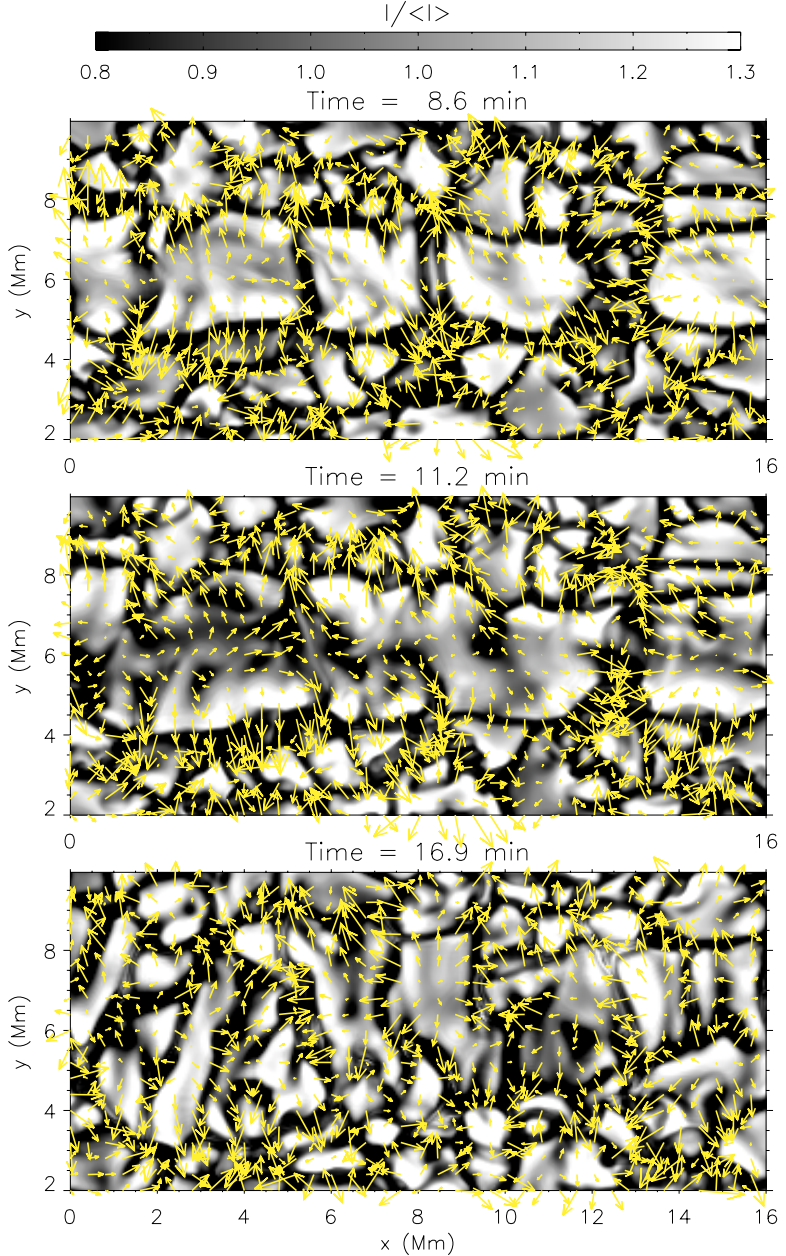

(a) Emergent intensity and horizontal flow field
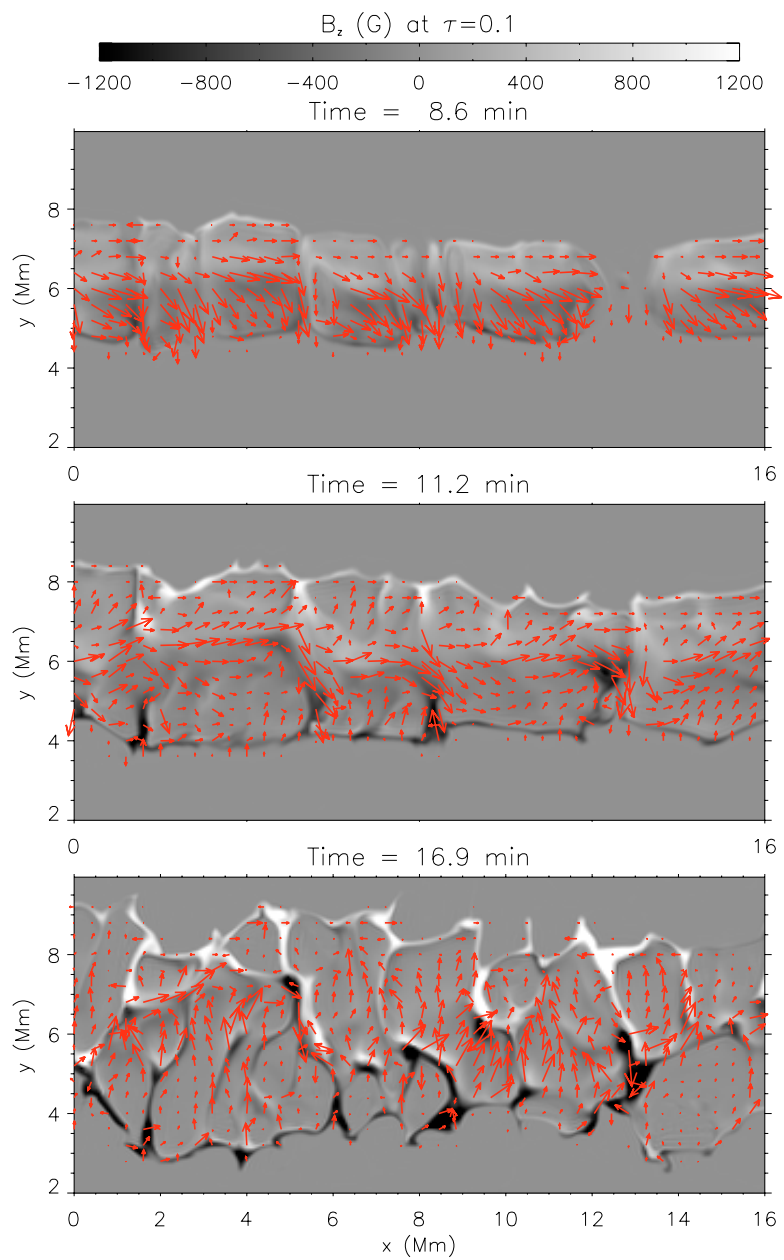

(b) Corresponding vector magnetograms

Fig. 13. Flux emergence event in run U1. Left: an arrow with a length of one grid-spacing on the vector plot corresponds to a horizontal flow speed of $v=3 \mathrm{~km} \mathrm{~s}^{-1}$. Right: an arrow with a length of one grid-spacing on the vector plot corresponds to a horizontal field strength of $|B|=400 \mathrm{G}$. Both the velocity and magnetic fields shown are evaluated at $\tau_{5000}=0.1$. The granulation pattern is temporarily disturbed by the emerging magnetic field and a transient darkening along the length of the domain marks the site of emergence. An accompanying mpeg animation is available.

maps for the same instant shows that much of the emerged flux already resides in intergranular lanes.

At $t=16.9 \mathrm{~min}$, the darkening has largely vanished and a granulation pattern more similar to the normal pattern has reestablished itself. However, these granules tend to be elongated in the $y$-direction because of the horizontal outflows driven by the expanding tube. In the corresponding magnetic field map, we find that the emerged magnetic flux outlines the boundaries of these new granules. The $y$-component of the magnetic field is, on average, positive. This is just the opposite to what we see in the magnetogram at $t=8.6 \mathrm{~min}$. This reversal of the sign of $B_{y}$ within this time span is expected. In the earlier snapshot, the $\tau_{5000}=0.1$ surface intersects with the upper half of the flux tube. The twist of the field lines within the tube is such that $B_{y}<0$ in this part of the tube. In the latter snapshot, the lower half of the tube has emerged at the surface, and so the synthetic magnetogram shows field with predominantly $B_{y}>0$.

\subsubsection{The relation between field strength and zenith angle}

Lites et al. (1998) have presented observational results for several emerging flux regions. They obtained vector magnetograms by applying inversion methods to measurements of the full
Stokes vector. Based on their observations, Lites et al. (1998) describe the following scenario for flux emergence: magnetic flux emerges at the surface in the form of horizontal field with field strengths of 200-600 G. After emergence, the field quickly migrates away from the emergence site. In the process, the field becomes vertical. Only then do the fields obtain field strengths exceeding one kilogauss. The observational study by Kubo et al. (2003) also supports these findings.

Figure 14 shows JPDFs of the zenith angle of the magnetic field vector versus the absolute field strength from simulation run U1. At $t=8.6 \mathrm{~min}$, the flux tube is just emerging at the surface. The JPDF at this time reflects the fact that the flux tube is still coherent. The horizontal fields are stronger than the vertical fields. Although a tiny fraction of the horizontal fields have kilogauss field strengths, most are confined within the range $400 \leq|B| \leq 1000 \mathrm{G}$. Just five minutes later, at $t=11.2 \mathrm{~min}$, the distribution in the JPDF looks very different. The appearance of two "horns" in the JPDF near $\gamma=0^{\circ}$ and $\gamma=180^{\circ}$ indicates the effect of the granular flow on the emerged field. As the emerged field is advected to the boundaries of granules, it also becomes predominantly vertical. As the simulation progresses in time, the two horns in the JPDF become more distinct. At $t=27.6 \mathrm{~min}$, nearly all the field with $|B| \geq 600 \mathrm{G}$ is vertical. There is 




Fig. 14. Joint probability distribution functions (JPDFs) of the zenith angle of the magnetic field vector versus the absolute field strength $|B|$ in run U1. Both quantities have been evaluated at the $\tau_{5000}=0.1$ level. A zenith angle of $90^{\circ}$ corresponds to a purely horizontal field. The four panels show the JPDFs at four different times. At $t=8.6 \mathrm{~min}$, the flux tube is emerging at the surface and the strongest fields are predominantly horizontal. Kilogauss fields develop only later and are almost exclusively vertical at $27.6 \mathrm{~min}$. actually an abundance of horizontal fields, but these are confined to strengths of less than $600 \mathrm{G}$. The shape of the JPDF at this time is similar to the scatter plots of zenith angle vs. $|B|$ obtained by Lites et al. (1998) and by Kubo et al. (2003).

\subsubsection{The dark lane}

The physical nature of the extended dark lane (see central panel of Fig. 13a) is distinctly different from that of normal intergranular lanes, which are associated with cold downflowing material. Instead, the dark lane that results from the emergence of the flux tube in run $\mathrm{U} 1$ is associated with upflowing material for a substantial fraction of its lifetime (several minutes). Figure 15 shows the normalized emergent continuum intensity at $t=11.2 \mathrm{~min}$ (same as the central panel in Fig. 10). Overplotted on the grey scale image are contours of the vertical component of the velocity at $\tau_{5000}=0.1$ for magnetic regions $(|B| \geq 400 \mathrm{G})$. The blue contours indicate upflows while the red contours correspond to downflows. There is a substantial overlap of the blue contours with regions on the surface comprising the dark lane. The correlation between upflows and low emergent intensity in the dark lane is also illustrated in Fig. 16, which shows profiles of emergent continuum intensity and vertical velocity at $\tau_{5000}=0.1$ across the dark lane (in the $y$-direction). The plotted values are averages taken along the $x$-direction at time $t=11.2 \mathrm{~min}$. Away from the emergence site, we have the usual intensity-velocity correlation of granules and intergranular lanes. However, within the emergence site (around $y \simeq 6 \mathrm{Mm}$ ), the darkening $\left(I_{5000} /\left\langle I_{5000}\right\rangle \approx 0.93\right)$ is co-spatial with upflowing material $\left(v_{z}=0.5-1 \mathrm{~km} \mathrm{~s}^{-1}\right)$.

The lifetime of the extended dark lane is about $10 \mathrm{~min}$, which is comparable to the timescale of granulation. During the first few minutes, the regions of lower brightness are associated with upflowing material with rise speeds of up to $1 \mathrm{~km} \mathrm{~s}^{-1}$. This is substantially lower than the typical rise speed $\left(v \approx 2-4 \mathrm{~km} \mathrm{~s}^{-1}\right)$ of magnetic material when it is just emerging at the visible surface. In the last few minutes of the dark lane's lifetime, the dark lane splits up into spatially separated, dark elongations with length of about $2 \mathrm{Mm}$ (see intensity image at $t=16.9 \mathrm{~min}$ in Fig. 13a). The vertical velocities of these dark elongations are negative, i.e., they have developed into downflows. The presence of the dark lane depends on the initial field strength as well as the initial twist carried by the tube. When the initial flux tube is sufficiently weak that its evolution is dominated by the drag force (as is the case with run U6), a dark lane does not appear: the magnetic flux is passively carried upward by the convective flow, which shows the normal intensity-velocity correlation. In order for the dark lane to appear, the emerging field must be strong enough that its evolution is not completely controlled by the convective flow. However, this is only a necessary criterion. The amount of initial twist is also important. We have carried out simulation runs with the same initial conditions for the flux tube as in run U1, varying only the amount of the initial twist. We found that for a flux tube with twist parameter $\lambda=0$, there is no transient dark lane marking the emergence site of the tube. For a flux tube with a moderate amount of twist, $\lambda=0.25$, we find the transient appearance of some dark patches within the granules where the emergence occurs. These patches, however, do not exhibit a clear alignment or coherence as is the case in run U6 (where $\lambda=0.5$ ). The following explanation accounts for the properties we have presented above: When a flux tube (with a flux of, say, $\Phi_{0}=10^{19} \mathrm{Mx}$ ) reaches the surface, it has a rise speed of $2-4 \mathrm{~km} \mathrm{~s}^{-1}$. As demonstrated by previous numerical MHD simulations of the rise of twisted flux tubes through an initially static atmosphere (see, for example, Moreno-Insertis \& Emonet 1996; Emonet \& Moreno-Insertis 1998; Cheung et al. 2006), the amount of twist in the tube determines whether it can remain a coherent structure (large $\lambda$ ) or break up into vortex fragments ( $\operatorname{small} \lambda)$. As the flux tube emerges and cools by radiation, it loses buoyancy. Nevertheless, the emerged magnetic material 


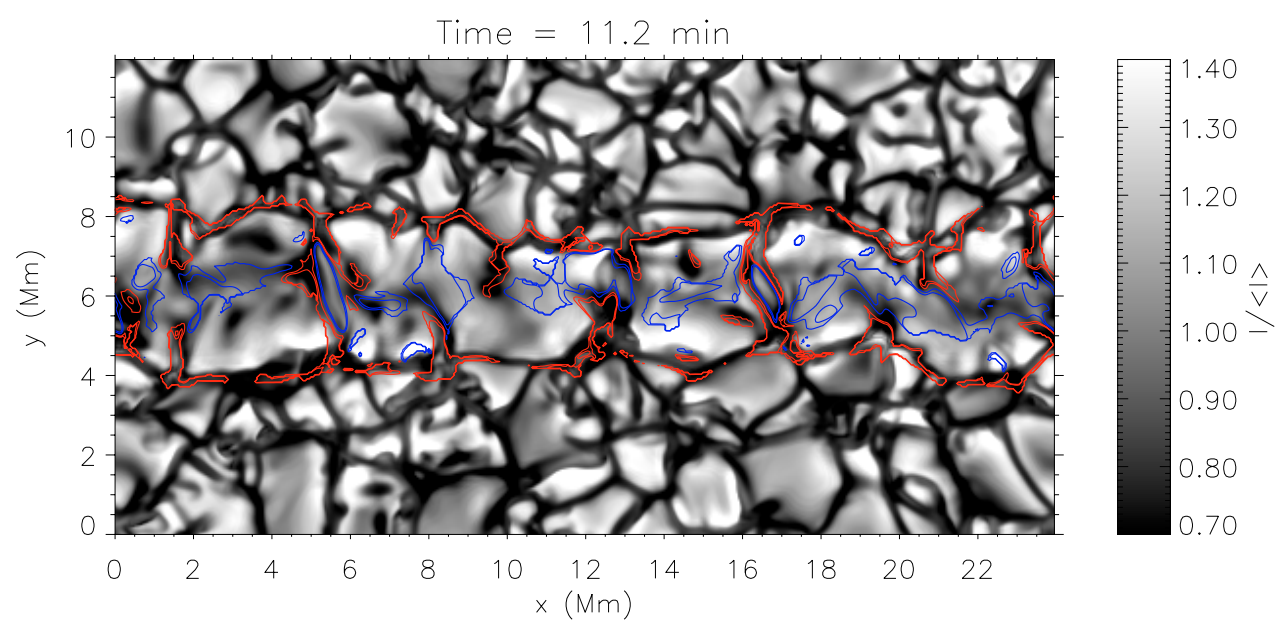

Fig. 15. Dark lane $(y \sim 6 \mathrm{Mm})$ in run $\mathrm{U} 1$ $(t=11.2 \mathrm{~min})$. The grey shading represents the normalized emergent continuum intensity. The blue contours indicate upflows with $v_{z}=0.5 \mathrm{~km} \mathrm{~s}^{-1}$ and $1 \mathrm{~km} \mathrm{~s}^{-1}$, respectively, while the red contours correspond to downflows of the same magnitude $\left(v_{z}<0\right)$. The dark lane is roughly coincident with upflowing material.

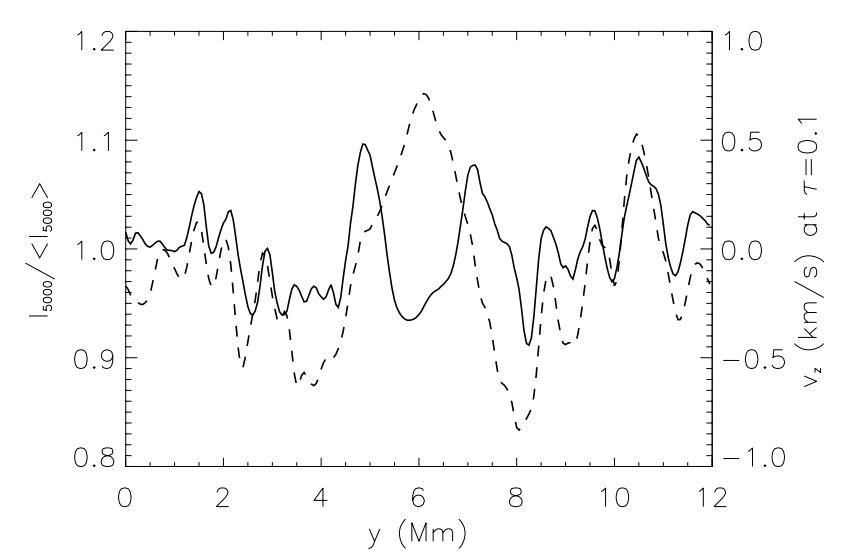

Fig. 16. Average profiles of the normalized continuum emergent intensity (solid line, left axis) and vertical velocity at $\tau_{5000}=0.1$ (dashed line, right axis) across the dark lane at $t=11.2 \mathrm{~min}$. The curves represent averages over the $x$-direction.

may still overshoot provided that the flux tube is sufficiently twisted, so that its core remains largely intact and maintains its magnetic buoyancy. As the emerged magnetic material of the tube overshoots, its upwards motion is braked by the stable stratification. This is why the upward velocities in Fig. 15 are limited to $0.5-1 \mathrm{~km} \mathrm{~s}^{-1}$ within the dark lane. The strong cooling of the magnetic material within the core of the tube at optical depth unity accounts for the lane's darkness. The arrangement of the cool and dense material in an extended lane configuration is not stable to perturbations introduced by granular dynamics. Within a few minutes, the extended dark lane breaks up into shorter elongations with lengths comparable to those of intergranular lanes. The buoyancy loss of the material by radiation and stratification either leads to the birth of new downflows, or feeds preexisting downflows in the intergranular network. The influence of the granular dynamics on its evolution explains why the dark lane has a lifetime comparable to the granulation timescale.

Transient appearance of dark alignments in emerging flux regions have been reported by a number of observational studies (Bray et al. 1984; Brants \& Steenbeek 1985; Zwaan 1985; Strous \& Zwaan 1999). For example, Strous \& Zwaan (1999) have carried out a statistical analysis of an emerging flux region and found that transient darkenings in the continuum as well as in the cores of photospheric spectral lines, lasting about $10 \mathrm{~min}$, are robust signatures of emergence events. They note that the typical extension of the darkenings is about $2 \mathrm{Mm}$ and that they are aligned roughly parallel to the direction connecting the two developing polarities of the corresponding active region. Strous \& Zwaan (1999) also report that the darkenings are associated with upflows of about $0.5 \mathrm{~km} \mathrm{~s}^{-1}$. These properties are reproduced by our simulation results from run U6. In addition, Strous \& Zwaan (1999) also report that the darkenings, on average, rotate counterclockwise at about $0.5^{\circ} \mathrm{min}^{-1}$. In run U6, we do find that some of the dark elongations rotate during the breakup of the dark lane. However, we do not find any systematic direction in which the rotation occurs.

\section{Discussion}

The magnetic flux emergence simulations presented in this paper are "realistic" in two senses. Firstly, the simulations take into account the effects of non-local radiative energy exchange, partial ionization and magneto-convection, all of which are important for a proper treatment of the problem. Secondly, the simulations yield observational signatures of magnetic flux emergence that are in qualitative and quantitative agreement with observations of emerging flux.

Our simulation results are generally consistent with the findings of Fan et al. (2003), extending their validity to the nearsurface layers, where the anelastic approximation used by these authors is no longer tenable. However, Mach numbers reaching $M \sim \mathrm{O}(0.1)-\mathrm{O}(1)$ and the steep decrease of pressure and density with height limit the range of possible ratios of initial and equipartition field strengths, $B_{0} / B_{\text {eq. }}$. As a consequence, a considerable influence of the convective flows on the dynamics of the rising flux tube and on the flux emergence process is unavoidable.

Convection influences the evolution of emerging magnetic fields both before and after they appear at the photosphere. In the subphotospheric layers, the convective flow distorts a rising flux tube and imposes a systematic undulation along the tube (with a typical wavelength $\sim 1-2 \mathrm{Mm}$ ). Whereas upflows aid the rise of certain segments of the tube, downflows suppress the rise of other segments. Our work shows that the undulatory nature of an emerging magnetic tube (Pariat et al. 2004) is a consequence of its interaction with granular convection.

The amount of distortion and deformation suffered by a magnetic flux tube interacting with convective flows depends on its initial properties, most notably its buoyancy and twist. Magnetic flux tubes with less longitudinal flux (say, $10^{18} \mathrm{Mx}$ ) are not sufficiently buoyant to rise coherently against the convective flows. The emergence events associated with these smaller and weaker 
flux tubes are inconspicuous, in the sense that the granulation pattern in the quiet Sun is not disturbed (see Sect. 4.1). Their small spatial scales $(\sim 1 \mathrm{Mm})$ and short temporal scales (5 min) make their observations difficult. Although detections of such events have been reported (De Pontieu 2002), it is unknown how much flux emerges at the surface in this form. High-cadence, high-resolution observations from the Solar Optical Telescope onboard Hinode (Solar-B, Ichimoto et al. 2004; Tarbell 2006) will allow us to study such events in more detail then ever before.

In Sect. 4.1.1, we provided an example of the secondary emergence of a bipole. This case (with a flux of $10^{18} \mathrm{Mx}$ in each polarity) is peculiar for two reasons. Firstly, the bipole emerged rather distant from the main emergence site. Secondly, it emerged several granulation time scales after the initial appearance of flux at the surface. This secondary emergence event is the result of the recirculation and overturning of material in the near-surface layers of the convection zone. It suggests to us that part of the small-scale flux emergence events on the solar surface could result from recirculation of material in the convection zone.

The expulsion of emerged magnetic flux from the granule interiors to the intergranular network on the order of the granulation time scale $(5-10 \mathrm{~min})$ is a common feature of all our flux emergence simulations. Having said this, not all emergence events appear the same. For example, the observational signatures of the emergence of tubes with $10^{19} \mathrm{Mx}$ flux are clearly different from those of tubes with $10^{18} \mathrm{Mx}$. Although they still suffer distortion and deformation by the convective flow, such bigger flux tubes are able to rise against downflows and eventually emerge at the surface. During emergence, the strong horizontal expansion of rising fluid can disturbs the granulation pattern. If the flux tube is sufficiently twisted (say, $\lambda=0.5$ ), the emergence event is accompanied by the transient appearance of an extended darkening in the direction of the tube axis. Such features have been reported in observations of emerging flux regions. Analysis of our simulations results reveals that they are associated with emerged, cooled magnetic material overshooting into the photosphere.

Acknowledgements. The authors thank the anonymous referee for helping to substantially improve the presentation of this paper. MCMC wishes to thank all the friends and colleagues at the IAC for their hospitality during his extended stays in Tenerife as a visiting graduate student. In particular, MCMC is deeply grateful to have known the late Juan Luis Medina Trujillo, who will always be remembered for his sincerity, his love of nature and his zest for life.

\section{References}

Abbett, W. P., \& Fisher, G. H. 2003, ApJ, 582, 475

Abbett, W. P., Fisher, G. H., \& Fan, Y. 2000, ApJ, 540, 548

Archontis, V., Moreno-Insertis, F., Galsgaard, K., Hood, A., \& O’Shea, E. 2004, ApJ, 441, 886

Archontis, V., Moreno-Insertis, F., Galsgaard, K., \& Hood, A. W. 2005, ApJ, 635, 1299

Bercik, D. J. 2002, Ph.D. Thesis, AA(Michigan State University)

Bercik, D. J., Nordlund, A., \& Stein, R. F. 2003, in ESA SP-517: GONG+ 2002, Local and Global Helioseismology: the Present and Future, ed. H. Sawaya-Lacoste (ESA Publications Division), 201

Born, R. 1974, Sol. Phys., 38, 127

Brants, J. J., \& Steenbeek, J. C. M. 1985, Sol. Phys., 96, 229

Bray, R. J., Loughhead, R. E., \& Durrant, C. J. 1984, The solar granulation (2nd edition) (Cambridge and New York: Cambridge University Press), 270

Caligari, P., Moreno-Insertis, F., \& Schüssler, M. 1995, ApJ, 441, 886

Caligari, P., Schüssler, M., \& Moreno-Insertis, F. 1998, ApJ, 502, 481

Cheung, M. 2006, Ph.D. Thesis, University of Göttingen, Germany, http://webdoc.sub.gwdg.de/diss/2006/cheung

Cheung, M. C. M., Moreno-Insertis, F., \& Schüssler, M. 2006, A\&A, 451, 303

Cheung, M. C. M., Schüssler, M., \& Moreno-Insertis, F. 2007, A\&A, 461, 1163

De Pontieu, B. 2002, ApJ, 569, 474
Deinzer, W., Hensler, G., Schüssler, M., \& Weisshaar, E. 1984, A\&A, 139, 426 Domínguez Cerdeña, I., Sánchez Almeida, J., \& Kneer, F. 2003, A\&A, 407, 741 Dorch, S. B. F., Gudiksen, B. V., Abbett, W. P., \& Nordlund, Å. 2001, A\&A, 380,734

D’Silva, S., \& Choudhuri, A. R. 1993, A\&A, 272, 621

Emonet, T., \& Moreno-Insertis, F. 1998, ApJ, 492, 804

Fan, Y. 2001, ApJ, 554, L111

Fan, Y., \& Gibson, S. E. 2004, ApJ, 609, 1123

Fan, Y., Fisher, G. H., \& Deluca, E. E. 1993, ApJ, 405, 390

Fan, Y., Fisher, G. H., \& McClymont, A. N. 1994, ApJ, 436, 907

Fan, Y., Zweibel, E. G., \& Lantz, S. R. 1998, ApJ, 493, 480

Fan, Y., Abbett, W. P., \& Fisher, G. H. 2003, ApJ, 582, 1206

Forbes, T. G., \& Priest, E. R. 1984, Sol. Phys., 94, 315

Galsgaard, K., Archontis, V., Moreno-Insertis, F., \& Hood, A. 2007, ApJ, submitted

Galsgaard, K., Moreno-Insertis, F., Archontis, V., \& Hood, A. 2005, ApJ, 618, L153

Grossmann-Doerth, U., Knölker, M., Schüssler, M., \& Solanki, S. K. 1994, A\&A, 285, 648

Grossmann-Doerth, U., Schüssler, M., \& Steiner, O. 1998, A\&A, 337, 928

Hagenaar, H. J. 2001, ApJ, 555, 448

Hagenaar, H. J., Schrijver, C. J., \& Title, A. M. 2003, ApJ, 584, 1107

Hurlburt, N. E., \& Toomre, J. 1988, ApJ, 327, 920

Ichimoto, K., Tsuneta, S., Suematsu, Y., et al. 2004, in Optical, Infrared, and Millimeter Space Telescopes, ed. J. C. Mather, Proc. SPIE, 5487, 1142

Isobe, H., Miyagoshi, T., Shibata, K., \& Yokoyama, T. 2005, Nature, 434, 478

Kubo, M., Shimizu, T., \& Lites, B. W. 2003, ApJ, 595, 465

Leake, J. E., \& Arber, T. D. 2006, A\&A, 450, 805

Lites, B. W., Leka, K. D., Skumanich, A., Martinez Pillet, V., \& Shimizu, T. 1996, ApJ, 460, 1019

Lites, B. W., Skumanich, A., \& Martinez Pillet, V. 1998, A\&A, 333, 1053

Longcope, D., Fisher, G., \& Arendt, S. 1996, ApJ, 464, 999

Magara, T. 2001, ApJ, 549, 608

Magara, T. 2006, ApJ, 653, 1499

Magara, T., \& Longcope, D. W. 2003, ApJ, 586, 630

Moreno-Insertis, F. 1983, A\&A, 122, 241

Moreno-Insertis, F., \& Emonet, T. 1996, ApJ, 472, L53

Murray, M. J., Hood, A. W., Moreno-Insertis, F., Galsgaard, K., \& Archontis, V. 2006, A\&A, 460, 909

Nordlund, A., \& Stein, R. F. 1990, in Solar Photosphere: Structure, Convection and Magnetic Fields, ed. J. O. Stenflo (Dordrecht: Kluwer Academic Publishers), IAU Symp., 138, 191

Pariat, E., Aulanier, G., Schmieder, B., et al. 2004, ApJ, 614, 1099

Parker, E. N. 1955, ApJ, 121, 491

Parker, E. N. 1979, ApJ, 230, 914

Proctor, M. R. E., \& Weiss, N. O. 1982, Reports of Progress in Physics, 45, 1317

Roberts, B., \& Webb, A. R. 1978, Sol. Phys., 56, 5

Schüssler, M. 1977, A\&A, 56, 439

Schüssler, M. 1979, A\&A, 71, 79

Schüssler, M. 1984, in The Hydromagnetics of the Sun (European Space Agency, ESA SP-220), 67

Schüssler, M. 1987, in The Internal Solar Angular Velocity, ed. B. R. Durney, \& S. Sofia, ASSL, 137, 303

Shibata, K., Tajima, T., Steinolfson, R. S., \& Matsumoto, R. 1989, ApJ, 345, 584 Solanki, S. K., Zufferey, D., Lin, H., Rueedi, I., \& Kuhn, J. R. 1996, A\&A, 310, L33

Spruit, H. C. 1976, Sol. Phys., 50, 269

Spruit, H. C. 1981, A\&A, 98, 155

Stein, R. F., \& Nordlund, A. 1989, ApJ, 342, L95

Stein, R. F., \& Nordlund, A. 1998, ApJ, 499, 914

Stein, R. F., \& Nordlund, A.. 2006, ApJ, 642, 1246

Steiner, O., Grossmann-Doerth, U., Knölker, M., \& Schüssler, M. 1998, ApJ, 495, 468

Strous, L. H., \& Zwaan, C. 1999, ApJ, 527, 435

Tarbell, T. D. 2006, in AAS/Solar Physics Division Meeting, 36.02

Title, A. M., Tarbell, T. D., Topka, K. P., et al. 1989, ApJ, 336, 475

Tobias, S. M., Brummell, N. H., Clune, T. L., \& Toomre, J. 2001, ApJ, 549, 1183

Török, T., \& Kliem, B. 2005, ApJ, 630, L97

Venkatakrishnan, P. 1986, Nature, 322, 156

Vögler, A. 2003, Ph.D. Thesis, University of Göttingen, Germany, http://webdoc.sub.gwdg.de/diss/2004/voegler

Vögler, A., Shelyag, S., Schüssler, M., et al. 2005, A\&A, 429, 335

Weiss, N. O. 1966, Royal Society of London Proceedings Series A, 293, 310

Zirin, H. 1972, Sol. Phys., 22, 34

Zwaan, C. 1978, Sol. Phys., 60, 213

Zwaan, C. 1985, Sol. Phys., 100, 397

Zwaan, C. 1987, ARA\&A, 25, 83 